\title{
ChemPer: An Open Source Tool for Automatically Generating SMIRKS Patterns
}

\author{
Caitlin C. Bannan, David Mobley
}

Submitted date: $21 / 06 / 2019$ - Posted date: 21/06/2019

Licence: CC BY 4.0

Citation information: Bannan, Caitlin C.; Mobley, David (2019): ChemPer: An Open Source Tool for Automatically Generating SMIRKS Patterns. ChemRxiv. Preprint.

Force fields are used in a variety of research fields including computer-aided drug design, biomaterials, and polymer chemistry. However, force fields also continue to limit the accuracy of predictions of physical properties. Current parameterization of these force fields involves a huge amount of human effort -- often years of work -- and depends heavily on the chemical intuition of those involved. The Open Force Field Initiative is working to replace this tedious process with an automated machinery to learn parameters and chemical perception. Our new SMIRKS-based force field format, SMIRNOFF, allows all parameter types to be defined independently. This allows for easier extension compared to the traditional atom type-based force fields where the chemical perception of all parameter types is intertwined. We will need to be capable of programmatically learning SMIRKS patterns in order to fully automate force field parameterization. In this work, we present ChemPer -- a new tool for generating SMIRKS patterns based on clustered fragments (i.e. bonds, angles, or torsions) which should be assigned the same force field parameter. We demonstrate the utility of ChemPer by clustering fragments based on a reference force field, and then regenerating those parameters starting with a simple set of alkanes, ethers, and alcohols. Next, we create SMIRKS patterns for a protein SMIRNOFF which match the parameters from AMBER99. We conclude with a discussion of other potential applications and expansions to ChemPer.

File list (3)

chemper.pdf (437.89 KiB)

view on ChemRxiv - download file

SI.pdf (141.56 KiB)

view on ChemRxiv - download file

electronic_SI.zip (46.32 MiB)

view on ChemRxiv - download file 


\title{
ChemPer: An Open Source tool for automatically generating SMIRKS patterns
}

\author{
Caitlin C. Bannan ${ }^{1}$ and David L. Mobley ${ }^{1,2}$ \\ ${ }^{1}$ Department of Chemistry, University of California, Irvine CA 92697; ${ }^{2}$ Department of \\ Pharmaceutical Science, University of California, Irvine CA 92697
}

*For correspondence: dmobley@mobleylab.org (DLM)

\begin{abstract}
Force fields are used in a variety of research fields including computer-aided drug design, biomaterials, and polymer chemistry. However, force fields also continue to limit the accuracy of predictions of physical properties. Current parameterization of these force fields involves a huge amount of human effort - often years of work - and depends heavily on the chemical intuition of those involved. The Open Force Field Initiative is working to replace this tedious process with an automated machinery to learn parameters and chemical perception. Our new SMIRKS-based force field format, SMIRNOFF, allows all parameter types to be defined independently. This allows for easier extension compared to the traditional atom type-based force fields where the chemical perception of all parameter types is intertwined. We will need to be capable of programmatically learning SMIRKS patterns in order to fully automate force field parameterization. In this work, we present ChemPer - a new tool for generating SMIRKS patterns based on clustered fragments (i.e. bonds, angles, or torsions) which should be assigned the same force field parameter. We demonstrate the utility of ChemPer by clustering fragments based on a reference force field, and then regenerating those parameters starting with a simple set of alkanes, ethers, and alcohols. Next, we create SMIRKS patterns for a protein SMIRNOFF which match the parameters from AMBER99. We conclude with a discussion of other potential applications and expansions to ChemPer.
\end{abstract}

\section{Introduction}

Molecular dynamics and Monte Carlo simulations are increasingly being used in a variety of fields from the study of materials and biopolymers to computer aided drug design [8, 9, 15, 28, 39, 44]. The accuracy of these simulations depends on the accuracy of the their potential function for calculation the energy and forces in the system. For these large systems, that potential function is typically a fixed charge, all atom force field which uses the positions of all atoms in the system to calculate the potential energy [40, 41]. While this is a simplistic representation, molecular simulations using force fields have shown promise in a variety of applications such as calculating hydration and solvation free energies $[22,36,45,46]$ as well as protein-ligand binding $[19,27,33,35]$. That being said, the literature is littered with examples of force field limitations $[5,6,11,13,16,17,21,24,26,29,30,33,42,47,51]$. Automating parameterization would allow tests of which functional forms result in the best and most general force fields [34].

Due to the considerable human intervention required, parameterizing a new small molecule force field can take decades $[20,50]$. Evaluating choices in functional form or reference data used in different force fields is nearly impossible because these choices cannot be separated from the human intuition of the group who did the parameterization. Chemical perception, the way a force field determines which parameters to assign to a molecule, remains the limiting step in automating force field parameterization. Traditionally, force fields in the AMBER [7, 50] and CHARMM [49] families have used indirect chemical perception in the form of 


\begin{tabular}{ll}
\hline Definition & Symbol \\
\hline any atom & $*$ \\
atomic number & $\# \mathrm{n}$ \\
aromatic or aliphatic & a or A \\
formal charge & $\pm \mathrm{n}$ \\
total hydrogen count & $\mathrm{Hn}$ \\
connectivity & $\mathrm{xn}$ \\
ring connectivity & $\mathrm{xn}$ \\
minimum ring size & $\mathrm{rn}$ \\
\hline
\end{tabular}

Table 1. Decorators used in SMIRKS patterns to describe atoms where $n$ represents an integer combined with any of those decorators.

\begin{tabular}{ll}
\hline Definition & Symbol \\
\hline any & $\sim$ \\
single & - \\
double & $=$ \\
triple & $\#$ \\
ring & $\mathbb{C}$ \\
\hline Table 2. Decorators used in SMIRKS patterns to describe bonds.
\end{tabular}

atom types to assign parameters. These atom types were assigned by hand making parameterization an extremely slow and tedious process.

The Open Force Field Initiative is working to automate force field parameterization in the new SMIRNOFF which uses SMIRKS patterns to assign parameters. SMIRKS is a substructure language derived from SMILES [12, 14]. They describe molecular fragments by logically combining decorators for atoms (Table 1 ) and bonds (Table 2). However, the SMIRKS patterns in current SMIRNOFFs have been written by hand. Automating force field parameterization in the SMIRNOFF format requires automatic creation of these patterns as a part of the process.

In this paper, we introduce ChemPer, a new tool for automatically determining SMIRKS patterns. Previous research has shown that generating SMIRKS patterns matching the chemical complexity of human derived chemical perception is a very difficult problem [52]. Therefore, in this work we will focus on SMIRKS generation assuming we already know which parameters we wish to assign to a set of molecules. That is the input for ChemPer modules are molecular fragments already clustered by which parameter they should be assigned. We validate these algorithms in two ways. First, we assign parameters to AlkEthOH [34] - a set of alkanes, ethers, and alcohols - with a reference force field and generate new SMIRKS to match that clustering. Second, we type a set of polypeptides with Amber99SB-ILDN [31] and attempt to generate SMIRKS patterns to recover these typing rules. These SMIRKS patterns could be used to create the first protein force field in the SMIRNOFF format. We conclude with a discussion of other possible applications and areas for potential improvement in the future. ChemPer is open source and available to the public at https://github.com/mobleylab/chemper [4].

\section{Motivation}

Recent attempts at sampling SMIRKS space highlights the difficulty in automating chemical perception. We introduced two tools, SMARTY and SMIRKY, which attempt to discover atom and fragment types using a Monte Carlo sampling algorithm. The foundational algorithm in these tools started from very generic patterns and created child parameters by making very simple changes to those patterns. Some downsides of this algorithm were straightforward, where perhaps a change in the type of moves could have lead to slightly higher success. However, the major takeaway, was the realization of the huge combinatorial problem when generating moves in SMIRKS space for parameter types with multiple atoms and bonds. In fact, we calculated that SMIRKY would take up to 1 billion moves to find SMIRKS patterns with the combination of decorators for certain torsions. To put this in perspective, a SMIRKY simulation of 100,000 iterations required over 700 CPU hours. This is largely due to the chemically naïve moves in SMIRKS space choosing new decorators for atoms and bonds at random with no concern for what combinations make chemical sense.

To make automated chemical perception truly feasible, we need to limit the search space by adding more chemical sense. However, this does not mean a human needs to encode rules for chemistry into the machinery; instead we should take advantage of the molecules being used to parameterize the force fields. A variety of cheminformatics tools are capable of extracting information about atoms and bonds in a molecule that we could convert into SMIRKS decorators [1,37]. This allows for a more deterministic approach 
where SMIRKS patterns are generated based on molecular fragments in the training set.

The Monte Carlo algorithm used in SMIRKY wasted computational time exploring SMIRKS space as a proxy for representing groups of fragments in training molecules. Testing typing of SMIRKS patterns is relatively fast; however, evaluating the accuracy of force field parameters requires much longer time lines. For example, evaluating new nonbonded parameters requires running molecular dynamics simulations to compare calculated and experimental physical properties such as densities of mixtures. [38]. Clustering molecular fragments based on moves in SMIRKS space could be the same as arbitrarily grouping those fragments before checking if they should receive the same parameter. It is possible there will be more robust mechanisms for grouping molecular fragments more directly, perhaps directly from quantum mechanical data. When parameterizing a general force field we need to generalize that clustering for reuse on different molecules. The SMIRKS patterns provide a mechanism for storing parameter assignment to be reused on new molecules.

An important underlying assumption in ChemPer is that the molecular fragments have already been clustered by force field parameter. In this work, we use established force fields to cluster molecular fragments and then generate SMIRKS for those clusters. For example, all bonds which are assigned the same force constant and equilibrium bond length could be clustered together. Determining the appropriate combination of clustering algorithms with ChemPer will require more research. For now, we focus on the problem of how to generate SMIRKS patterns for groups of fragments.

ChemPer must generate hierarchical SMIRKS for use with SMIRNOFF parameters. The SMIRNOFF format uses a "last one wins" strategy where the last SMIRKS pattern to match a set of atoms determines the parameter assigned. Imagine we have two nonbonded parameters for hydrogen atoms: $\mathrm{n} 1$ (" [\#1:1]"), which matches any hydrogen atom, and n2 ("[\#1:]-[\#8]") for any hydrogen atom bonded to oxygen. Initially the SMIRKS for $\mathrm{n} 1$ would match all hydrogens, but then the hydrogens bound to oxygen would match and be assigned the $\mathrm{n} 2$ parameter. The central goal of ChemPer is to start with clusters of molecular fragments where each fragment in a cluster should be assigned the same force field parameter and end with a hierarchical list of SMIRKS patterns which maintain the provided clustering.

\section{Methods}

ChemPer was created to generate hierarchical SMIRKS patterns. These can be used when parameterizing SMIRNOFFs. We assume the SMIRKS patterns will be applied in order, that is the last pattern to match a fragment will determine which force field parameter will be assigned. Starting with molecular fragments, ChemPer generates SMIRKS in a series of steps The primary input for ChemPer is clustered molecular fragments. This requires a series of internal steps which are described in detail in the subsections below. Wrappers around established cheminformatics toolkits are used to extract atom and bond properties corresponding to SMIRKS decorators (Section 3.1). Atoms and bonds are stored in a graph in order to track which SMIRKS decorators could be applied to each (Sections 3.2 and 3.3). SMIRKS are generated for each input cluster of molecular fragments storing all possible decorators (Section 3.4). More general patterns are created by stochastically removing decorators from atoms and bonds while maintaining the input clusters (Section 3.5). This modular arrangement will allow for experimentation in the clustering and ordering of molecular fragments during force field parameterization.

\subsection{ChemPer wraps around established toolkits}

ChemPer creates SMIRKS patterns based on molecules in a training set, meaning it depends on cheminformatics toolkits to access properties of atoms and bonds in those molecules. Specifically, OpenEye toolkits [32, 37, 48] or the RDKit [1] packages are used to access information about the atoms and bonds used when creating SMIRKS patterns. The mol_toolkit module includes three classes, Mol, Atom, and Bond which wrap around the corresponding molecule, atom, and bond objects in a dependent toolkit. These wrappers allow a user access to the rest of ChemPer tools without specifying which toolkit they are using. This setup allows for future support of other cheminformatics toolkits assuming the functions detailed below can be created. 
ChemPer Atom and Bond objects include functions related to the properties and local connections of these objects. They can be used to extract properties corresponding to SMIRKS decorators for atoms (Table 1) and bonds (Table 2). Both allow access to the molecule they came from. Atom objects also have functions to check if they are bonded to another atom and to identify neighboring atoms or bonds. Bond objects include access to the atoms it connects.

Mol objects include common functions for molecules, including access to atoms and bonds. A user can iterate over the atoms and bonds in the molecule or extract them based on a provided index. A bond can be identified by providing two atoms; if the atoms are not connected then None is returned instead. A Mol can be created from a SMILES string and can create a SMILES. Mol includes substructure searches in order to find atoms matching a provided SMIRKS pattern.

\subsection{Bespoke SMIRKS can be created with SingleGraph objects}

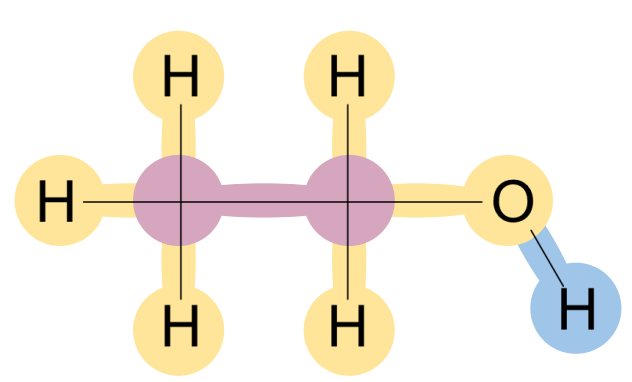

Figure 1. A demonstration of how the SingleGraph module generates SMIRKS. The user provides a molecule object, in this case ethanol, and specifies which atoms should be tagged (pink). The number of "layers" determines how many non-indexed atoms should be included. With layers $=1$, the atoms one bond away (yellow) are included. To include al atoms in a molecule, adding the blue ones in this example, layers can be set to \}all.' The SMIRKS, shown in the table at the right, can include all decorators (complex) or just the atomic numbers (compressed).

\begin{tabular}{|c|}
\hline layers $=0$ \\
\hline Compressed SMIRKS: “[\#6:1]-; !@ [\#6:2]” \\
\hline Complex SMIRKS: “[\#6AH3X4x0!r+0:1] \\
\hline$-; ! @[\# 6 A H 2 X 4 x 0 ! r+0: 2]^{\prime \prime}$ \\
\hline layers $=1$ \\
\hline Compressed SMIRKS: “[\#6:1] (-;!@[\#1]) \\
\hline$(-; ! @[\# 1])(-; ! @[\# 1])-; ! @[\# 6: 2]$ \\
\hline$(-; ! @[\# 1])(-; ! @[\# 1])-; ! @[\# 8]^{\prime \prime}$ \\
\hline Complex SMIRKS: “[\#6AH3X4x0!r+0:1] \\
\hline$(-; ! @[\# 1 \mathrm{AHOX} 1 \mathrm{x} 0 ! \mathrm{r}+0])(-; ! @[\# 1 \mathrm{AHOX} 1 \mathrm{x} 0$ \\
\hline$! r+0])(-; ! @[\# 1 \mathrm{AH} 0 \mathrm{X} 1 \mathrm{x} 0 ! \mathrm{r}+0])-; ! @[\# 6 \mathrm{AH} 2$ \\
\hline $\mathrm{X} 4 \mathrm{x} 0 ! r+0: 2](-; ! @[\# 1 \mathrm{AH} 0 \mathrm{X} 1 \mathrm{x} 0 ! r+0])(-; ! @$ \\
\hline$[\# 1 \mathrm{AH} 0 \mathrm{X} 1 \mathrm{x} 0 ! \mathrm{r}+0])-; ! @[\# 8 \mathrm{AH} 1 \mathrm{X} 2 \mathrm{x} 0 ! \mathrm{r}+0] "$ \\
\hline layers='all' \\
\hline Compressed SMIRKS: “[\#6:1] (-;!@[\#1]) \\
\hline$(-; ! @[\# 1])(-; ! @[\# 1])-; ! @[\# 6: 2]$ \\
\hline$(-; ! @[\# 1])(-; ! @[\# 1])-; ! @[\# 8]-; ! @[\# 1]^{\prime \prime}$ \\
\hline Complex SMIRKS: “[\#6AH3X4x0!r+0:1] \\
\hline$(-; ! @[\# 1 \mathrm{AHOX} 1 \mathrm{x} 0 ! \mathrm{r}+0])(-; ! @[\# 1 \mathrm{AHOX} 1 \mathrm{x} 0$ \\
\hline$! r+0])(-; ! @[\# 1 \mathrm{AH} 0 \mathrm{X} 1 \times 0 ! r+0])-; ! @[\# 6 \mathrm{AH} 2$ \\
\hline $\mathrm{X} 4 \mathrm{x} 0 ! \mathrm{r}+0: 2](-; ! @[\# 1 \mathrm{AH} 0 \mathrm{X} 1 \mathrm{x} 0 ! r+0])(-; ! @$ \\
\hline$[\# 1 \mathrm{AH} 0 \mathrm{X} 1 \mathrm{x} 0 ! \mathrm{r}+0])$ \\
\hline$-; ! @[\# 8 \mathrm{AH} 1 \mathrm{X} 2 \mathrm{x} 0 ! \mathrm{r}+0]-; ! @[\# 1 \mathrm{AHOX} 1 \mathrm{x} 0 ! \mathrm{r}+0] "$ \\
\hline
\end{tabular}

The SingleGraph module provides a foundation for the more complex manipulations required to make SMIRKS for groups of fragments. SingleGraph is used to generate a SMIRKS pattern for one fragment in a single molecule (Figure 1). SingleGraph is initiated with three inputs from the user:

1. a molecule from any of the supported toolkits,

2. the index of atoms which should be tagged in the SMIRKS pattern, and

3. the number of "layers."

Layers indicates how many non-tagged atoms should also be included in the SMIRKS pattern. This is an integer describing how many bonds away from the tagged atoms should be considered, or it can be the string "all" indicating that all atoms in the molecule should be used in the SMIRKS. A NetworkX [18] graph is used to store decorators for atoms (nodes) connected by bonds (edges). Then the SMIRKS can be created 
for this graph in two forms. First, the complex SMIRKS include all decorators for every atom. Second, the compressed SMIRKS include only the atomic number for atoms, leaving off other decorators (Table 1). In both cases, bonds include decorators specifying their order and whether they are a part of a ring. Below is an example implementation of this module.

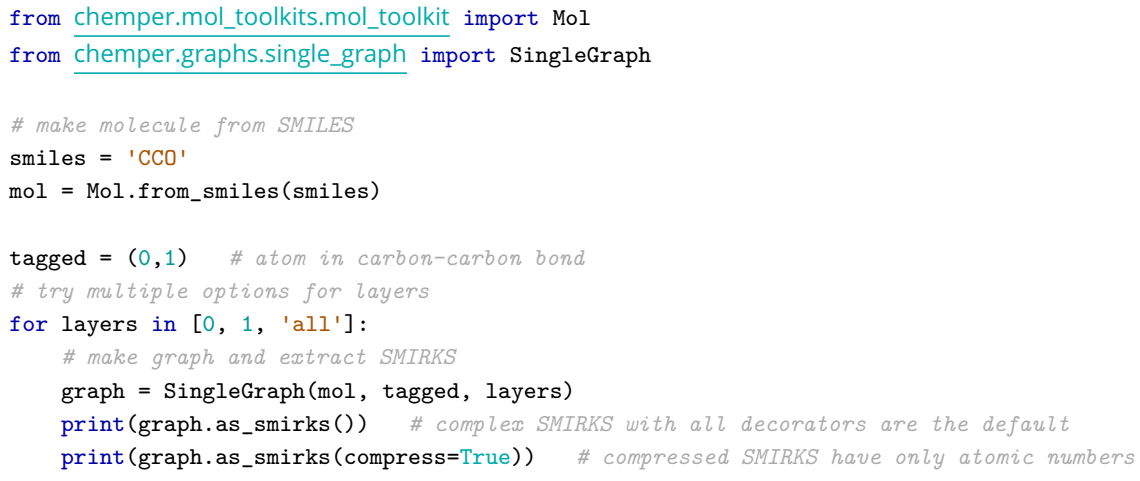

To further illustrate SingleGraph, consider the example in Figure 1 where we create SMIRKS for the carbon-carbon bond in ethanol. This is implemented in the code snippet above. The molecule object can be created from a SMILES (' $\mathrm{CC}$ '), where the carbons are at index 0 and 1 . We then decide how many layers to include. Zero layers would only include the carbon-carbon bond, then one layer would add the hydrogens and oxygen immediately bonded to those carbons. If we specify two layers the hydrogen in the alcohol group would also be added; for ethanol this is equivalent to specifying 'all' atoms. Then we can output the graph as a complex SMIRKS pattern (i.e. [\#6H3X4x0!r+0A:1]-; ! @ [\#6H2X4x0!r+0A:2]) or in the compressed form with only atomic numbers (i.e. [\#6:1]-;@[\#6:2]).

\subsection{ClusterGraph objects combine decorators from multiple fragments into one SMIRKS}

The ClusterGraph object expands on the SingleGraph idea by creating SMIRKS for groups of molecular fragments. The underlying structure is the same with a graph connecting atoms (nodes) by bonds (edges). As depicted in the implementation example below, the inputs also mirror SingleGraph, but it takes a list of molecules instead of a single molecule. The atoms are specified by lists of indices that should be included in the SMIRKS for each molecule. To illustrate the differences we will consider making SMIRKS which match the carbon-carbon single bonds in ethanol and propene (Figure 2). The SMIRKS for this example can be generated with the code snippet below.

ClusterGraph nodes must store all possible combinations of decorators for atoms and the edges do the same for all bonds.

This is done by storing sets of decorators. If multiple atoms have the same combination of decorators then that set of decorators will only be stored once. In the example, the SMIRKS match two different carbon-carbon bonds, however the first tagged atoms (:1) only has one set of decorators ("[\#6H3X4x0!r+OA:1]") which work for both fragments. However, the second carbon has two different combinations of decorators which

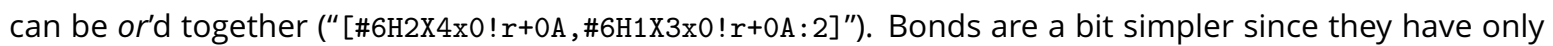
two types of decorators: (1) bond order and (2) if it is in a ring. The orders can be or'd together, similar to atom decorators. The bond,$-=;$ !@ is added when the layers variable is greater than zero. It matches the carbon-oxygen bond in ethanol and the carbon-carbon double bond in propene which means that bond can be single (-) or double (=), but is always not in a ring (! $\odot)$. The $@$ decorator for bonds is only used if all bonds in that cluster are either in a ring (৫) or not in a ring (! ৫).

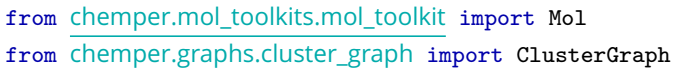




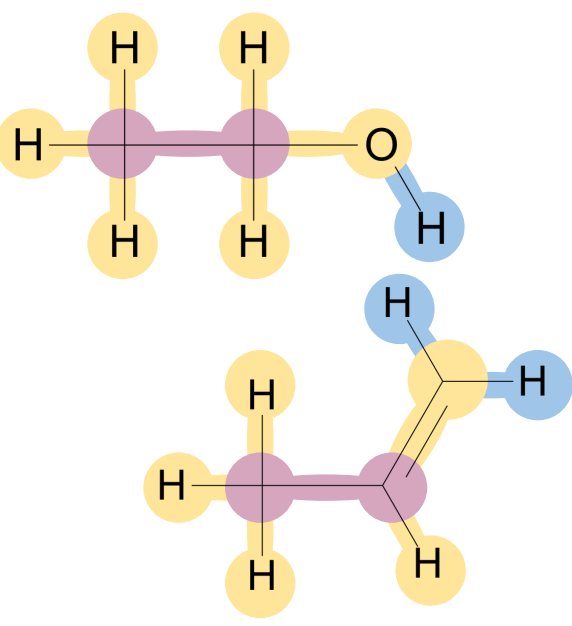

Figure 2. A demonstration of how the ClusterGraph module generates SMIRKS. The user provides a list of molecule objects, in this case ethanol and propene, and specifies which atoms should be tagged (pink). The number of "layers" determines how many how many nonindexed atoms should be included. With layers $=1$, the atoms one bond away (yellow) are included. To include all atoms in a molecule, adding the blue ones in this example, layers can be set to 'all.' The complex SMIRKS have all possible combinations of decorators or'd (,) together. In the compressed SMIRKS decorators which are in all of the combinations are and'd (;) to the end of each atom.

\begin{tabular}{|c|}
\hline layers $=0$ \\
\hline $\begin{array}{l}\text { Compressed SMIRKS: “[\#6H3X4x0!r+0A:1] } \\
-; \text { ! @ [\#6H1X3,\#6H2X4;!r;+0; } 0 ; \mathrm{A}: 2] "\end{array}$ \\
\hline $\begin{array}{l}\text { Complex SMIRKS: “[\#6H3X4x0!r+0A: 1] } \\
-; ! @[\# 6 \mathrm{H} 1 \mathrm{X} 3 \mathrm{x} 0 ! \mathrm{r}+0 \mathrm{~A}, \# 6 \mathrm{H} 2 \mathrm{X} 4 \mathrm{x} 0 ! \mathrm{r}+0 \mathrm{~A}: 2] "\end{array}$ \\
\hline layers=1 \\
\hline 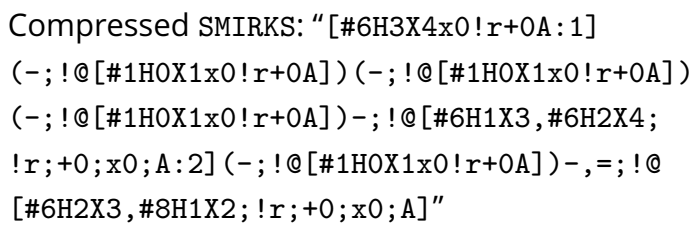 \\
\hline $\begin{array}{l}\text { Complex SMIRKS: “[\#6H3X4x0!r+0A : 1] } \\
(-; ! @[\# 1 \mathrm{HOX} 1 \mathrm{x} 0 ! \mathrm{r}+0 \mathrm{~A}])(-; ! @[\# 1 \mathrm{H} 0 \mathrm{X} 1 \mathrm{x} 0 ! \mathrm{r}+0 \mathrm{~A}]) \\
(-; ! @[\# 1 \mathrm{H} 0 \mathrm{X} 1 \mathrm{x} 0 ! \mathrm{r}+0 \mathrm{~A}])-; ! @[\# 6 \mathrm{H} 1 \mathrm{X} 3 \mathrm{x} 0 ! \mathrm{r}+0 \mathrm{~A}, \\
\# 6 \mathrm{H} 2 \mathrm{X} 4 \mathrm{x} 0 ! \mathrm{r}+0 \mathrm{~A}: 2](-; ! @[\# 1 \mathrm{H} 0 \mathrm{X} 1 \mathrm{x} 0 ! \mathrm{r}+0 \mathrm{~A}]) \\
-,=; ! @[\# 6 \mathrm{H} 2 \mathrm{X} 3 \mathrm{x} 0 ! \mathrm{r}+0 \mathrm{~A}, \# 8 \mathrm{H} 1 \mathrm{X} 2 \mathrm{x} 0 ! \mathrm{r}+0 \mathrm{~A}] ”\end{array}$ \\
\hline layers=‘all' \\
\hline 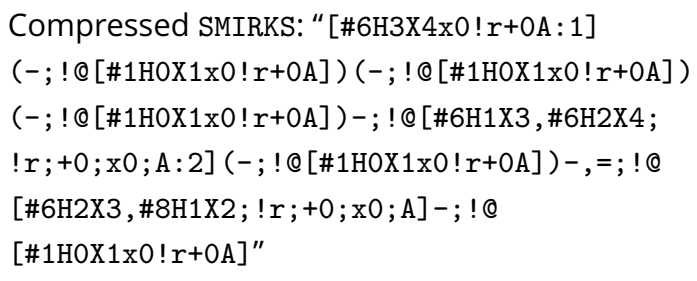 \\
\hline 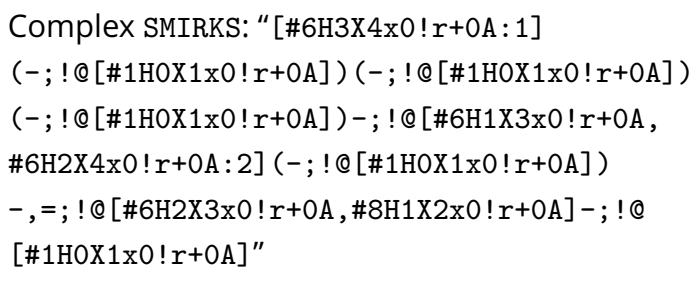 \\
\hline
\end{tabular}

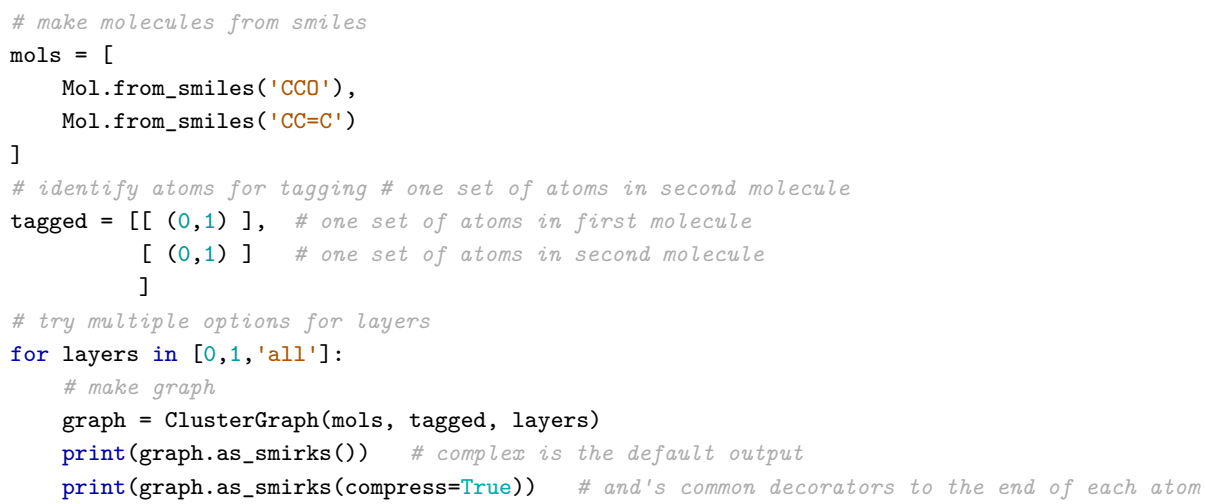

ClusterGraphs can be written out as complex or compressed SMIRKS.

In this case, a compressed SMIRKS retains decorators on atoms, but they are arranged differently. The atoms store all combinations of decorators, however it is also useful to know which decorators are always included on an atom. In the compressed SMIRKS, decorators, which atoms from all molecules have in common, 
are and'd (;) to the end of that SMIRKS. Consider again atom 2 in the ethanol/propene example. Atom 2 occurs in two different environments, but these two cases share some decorators. In the complex SMIRKS (" $[\# 6 \mathrm{H} 2 \mathrm{X} 4 \mathrm{xO} 0$ !r $+0 \mathrm{~A}, \# 6 \mathrm{H} 1 \mathrm{X} 3 \times \mathrm{x} 0$ ! $\mathrm{r}+\mathrm{OA}: 2]$ ") all decorators are treated separately, but in the compressed SMIRKS (" $[\# 6 \mathrm{H} 2 \mathrm{X} 4, \# 6 \mathrm{H} 1 \mathrm{X} 3 ; ! \mathrm{r} ;+0 ; \mathrm{x} 0 ; \mathrm{A}: 2]$ ") the common decorators are and'd to the end. This is useful when trying to isolate which decorators are important for telling one cluster apart from another.

\section{Creating SMIRKS for clusters requires properly aligning atoms.}

It does not matter which order atoms are given to ClusterGraph. They are rearranged so the most similar combination of decorators is put into the same atom. In SMIRNOFF, when a SMIRKS pattern is matched to a molecule it doesn't matter which atom is tagged with which number. In a bond parameter it does not matter which atom is tagged as : 1 or :2 because the parameter is assigned to the bond between those atoms. For example, if we wanted a parameter for a carbon-nitrogen single bond we could right "[\#6:1]-[\#7:2]" or "[\#7:1]-[\#6:2]" and the parameter would be assigned to the same bond. Therefore, we want a SMIRKS which aligns fragments so the most similar atoms are combined into the same node. To accomplish this we compare the SMIRKS decorators for incoming atoms with those already stored in the graph. In the ethanol/propene example, the carbon-carbon bond in ethanol is added first making the SMIRKS pattern " $[\# 6 \mathrm{H} 3 \times 4 \times \mathrm{x} 0 ! \mathrm{r}+0 \mathrm{~A}: 1]-; ! @[\# 6 \mathrm{H} 2 \times 4 \times \mathrm{x} 0 ! \mathrm{r}+0 \mathrm{~A}: 2]$." Then, the decorators for the two carbons in propene

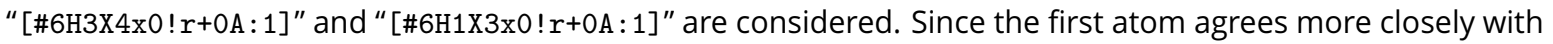
atom $: 1$ the propene atoms are added in order. However, if the user provided the atoms backwards $((1,0)$ instead of $(0,1))$ then we would invert the sub-graph so the atoms are added to the node they most closely align with. This process iterates over the layers of untagged atoms as well to make sure they align in a logical fashion.

\subsection{The SMIRKSifier creates a hierarchical list of SMIRKS from input molecules}

The central tool in ChemPer is the SMIRKSifier which builds on the ClusterGraph to generate a list of SMIRKS patterns based on provided clusters of molecular fragments. We will return to the ethanol/propene example used above, but this time there are six clusters based on the typical bond stretch force field parameters, as illustrated in Figure 3. The implementation of SMIRKSifier to make these SMIRKS patterns is included below. Like the graph modules described above, SMIRKSifier is initiated with a list of molecules and atoms in each cluster are specified by index. Internally, a clusterGraph is created for each provided cluster starting with zero layers. Then, we check to see if the SMIRKS patterns maintain the provided clustering using a scoring function based on our previously reported SMIRKY algorithm (Section 3.4.1) [52]. Additional layers are added iteratively until a set of SMIRKS accurately reproduces the provided clustering or reaches a user specified maximum number of layers (default=5). If such SMIRKS cannot be created, for example if chemically identical fragments are placed in separate clusters, an exception is raised. Once a successful list of SMIRKS is created, SMIRKSifier interfaces with the Reducer to remove unnecessary decorators throughout the patterns (Section 3.5). Both SMIRKSifer and Reducer have "verbose" as an optional variable to print out information about the process of SMIRKS creation and reduction.

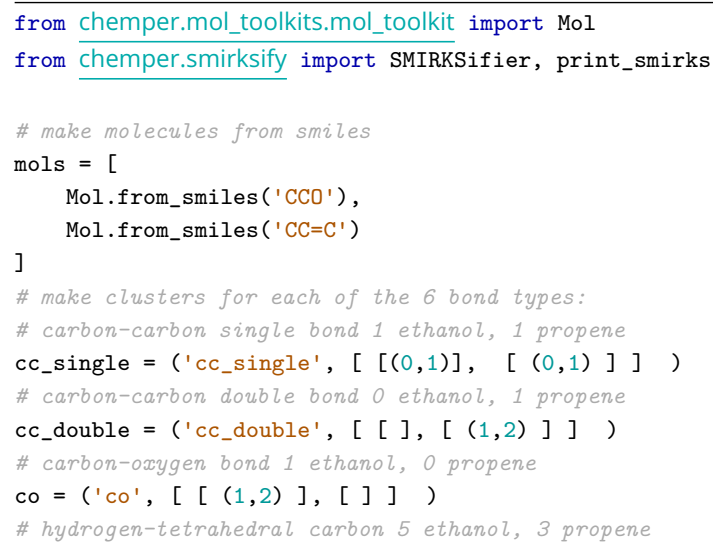



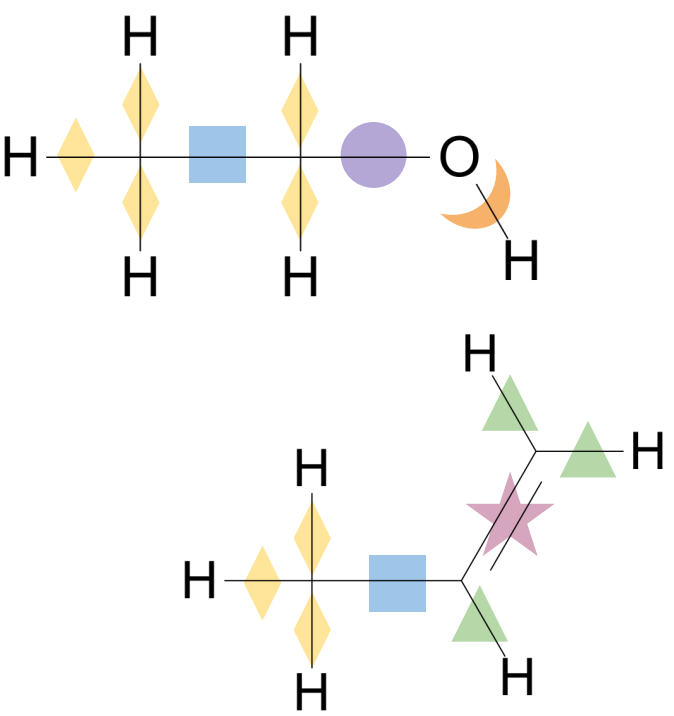

Figure 3. A demonstration of how SMIRKSif ier creates hierarchical SMIRKS patterns for provided molecular fragments. Each bond in these molecules is labeled by a symbol corresponding to it assigned cluster: carbon-carbon single (blue squares), carbon-carbon double (pink star), carbon-oxygen single (purple circle), hydrogen-tetrahedral carbon (yellow diamond), hydrogen-trivalent carbon (green triangle), and hydrogen-oxygen (orange crescent). The initial generated SMIRKS patterns and the reduced form are included on the left with their corresponding symbol.

\begin{tabular}{|c|}
\hline Initial SMIRKS \\
\hline [\#6H3X4x0!r+0A:1]-;!@ \\
\hline$[\# 6 \mathrm{H} 1 \mathrm{X} 3, \# 6 \mathrm{H} 2 \mathrm{X} 4 ; ! \mathrm{r} ;+0 ; \mathrm{x} 0 ; \mathrm{A}: 2]$ \\
\hline$[\# 6 \mathrm{H} 1 \mathrm{X} 3 \mathrm{x} 0 ! \mathrm{r}+\mathrm{OA}: 1]=; ! @$ \\
\hline$[\# 6 \mathrm{H} 2 \times 3 \times \mathrm{xk} 0 ! \mathrm{r}+0 \mathrm{~A}: 2]$ \\
\hline$[\# 6 \mathrm{H} 2 \mathrm{X} 4 \mathrm{x} 0 ! \mathrm{r}+0 \mathrm{~A}: 1]-; ! @$ \\
\hline$[\# 8 \mathrm{H} 1 \mathrm{X} 2 \mathrm{x} 0 ! \mathrm{r}+0 \mathrm{~A}: 2]$ \\
\hline$[\# 6 \mathrm{H} 2, \# 6 \mathrm{H} 3 ; ! \mathrm{r} ;+0 ; \mathrm{X} 3 ; \mathrm{x} 0 ; \mathrm{A}: 1]$ \\
\hline -; !@ [\#1HOX1x0!r+0A:2] \\
\hline$[\# 6 \mathrm{H} 1, \# 6 \mathrm{H} 2 ; ! r ;+0 ; \mathrm{X} 3 ; \mathrm{x} 0 ; \mathrm{A}: 1]$ \\
\hline$[-; ! @ \# 1 \mathrm{HOX} 1 \mathrm{x} 0 ! \mathrm{r}+0 \mathrm{~A}: 2]$ \\
\hline$[\# 8 \mathrm{H} 1 \mathrm{X} 2 \mathrm{x} 0 ! \mathrm{r}+0 \mathrm{~A}: 1]-; ! @$ \\
\hline$[\# 1 \mathrm{H} 0 \mathrm{X} 1 \mathrm{x} 0 ! \mathrm{r}+0 \mathrm{~A}: 2]$ \\
\hline Reduced SMIRKS \\
\hline$[*: 1] \sim[*: 2]$ \\
\hline$[*: 1] \sim[\# 6 \mathrm{H} 2 \mathrm{X} 3: 2]$ \\
\hline$[*: 1] \sim[\# 8: 2]$ \\
\hline$[*: 1] \sim[\# 1: 2]$ \\
\hline$[* ; \mathrm{X} 3: 1] \sim[\# 1: 2]$ \\
\hline$[\# 8: 1] \sim[\# 1: 2]$ \\
\hline
\end{tabular}

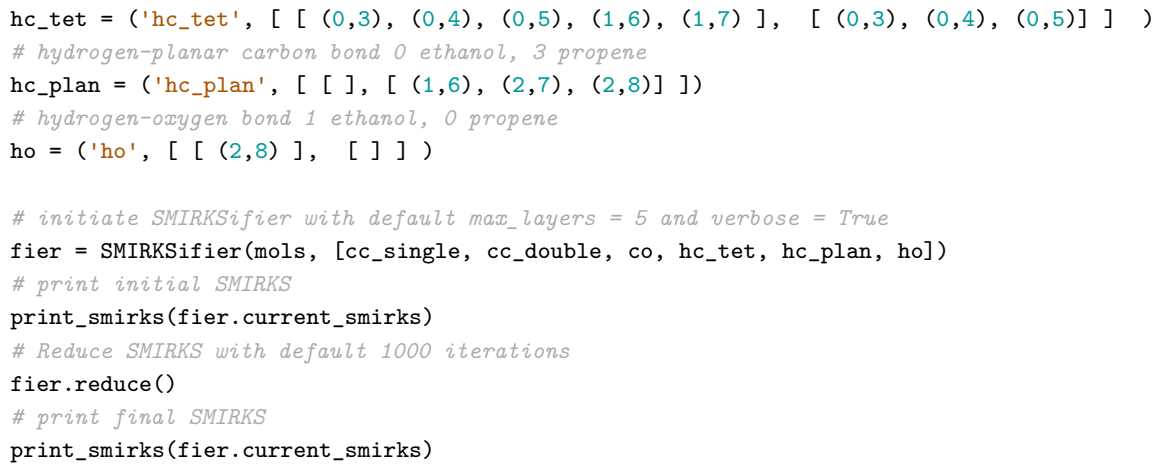

\subsubsection{The SMIRKSifier criteria evaluates SMIRKS patterns using a bipartite graph.}

To determine if we have added a sufficient number of layers, we need to check if the generated SMIRKS patterns match the input clusters. This approach utilizes a simplified version of the scoring function developed for SMIRKY. Each fragment (i.e. bond) has two types, the current type assigned by the SMIRKS patterns and a reference type based on its input cluster. Using NetworkX, a bipartite graph is created where each current type has a node on side $\mathbf{A}$ and each reference type has a node on side $\mathbf{B}$. For every fragment in every molecule we draw an edge connecting its current type to its reference type. In ChemPer we do not 
allow partial matches between the current and reference types. That means if any node has more than one edge the current list of SMIRKS pattern is rejected because it does not match the input clusters.

\subsection{Reducer stochastically removes decorators from SMIRKS patterns}

We created the Reducer to make SMIRKS patterns more general by removing unnecessary decorators. The goal of ChemPer is to create SMIRKS patterns for general SMIRNOFF force fields which means we want SMIRKS that will keep our desired clustering in molecules not used in the training set. Thus, we cannot store the ClusterGraph created SMIRKS which specify all possible decorators. This is a standalone class which is initiated from a list of SMIRKS patterns and training molecules. The Reducer takes a set number of iterations to stochastically simplify SMIRKS patterns by attempting to remove decorators. Each iteration uses the following steps:

1. choose one SMIRKS pattern from the current set to change,

2. choose an atom or bond to change,

3. make one change to that atom or bond, and

4. decide to accept or reject the change based on the SMIRKSifier criteria.

In Step 1, SMIRKS from the current list is chosen completely randomly. Steps 2 and 3 take advantage of ChemicalEnvironments to break SMIRKS into their atom and bond components and then edit the decorators within. The final step (4) uses the same approach described above (Section 3.4.1).

Choosing which component of a SMIRKS to remove decorators from is weighted by their current complexity. First, a ChemicalEnvironment is created from the chosen SMIRKS pattern which allows us to loop over all of its atoms and bonds. For each component (atom or bond), we store the number of decorators in that component. Finally, a component to change is chosen at random weighted by the number of decorators it contains. This means that an "empty" atom or bond, one with no decorators, will never be chosen, if all of the atoms and bonds are already empty then this iteration is rejected with no changes made.

There are a variety of ways decorators can be removed from a SMIRKS pattern. The second step, after choosing an atom or bond, is to chose which category of removal type will be performed. The three category types are

a) removal of an untagged atom that has only one neighbor,

b) changes to decorators and'd to the end of an atom or bond, and

c) changes to or'd together decorators.

The category is chosen at random, but only if it is possible in the current SMIRKS pattern. For example, an atom can only be removed if it is untagged (does not have $a: n$ in the SMIRKS) and is only connected to one other atom. Therefore, if a tagged atom, an atom with two neighbors, or a bond was chosen, this category would not be available in the random decision. Similarly, if the component has no or'd together decorators this option would not be available. If option a) is chosen the atom and corresponding bond are removed. Currently in ChemPer bonds can only have one and'd decorator either in a ring (@) or not in a ring (! $)$. Thus, for bonds option b) means removing whichever option is there. In a bond the or'd decorators correspond to bond orders. Removing a single bond order would lead to that bond not matching all bonds in its cluster, so for a bond in option c) all or'd decorators are removed.

For atoms, there are subcategories for options b) or c) to chose from (Table 3). We will use the atom "[\#6Х3Н $2, \# 7 \times 2 \mathrm{H} 1 ;+0 ; \mathrm{A}]$ " as an example of how decorators can be removed. For and'd decorators, we chose randomly with equal probability to either remove one (either ' $+O{ }^{\prime}$ or ' $A$ ') or all of the decorators. If one decorator is to be removed, that one is chosen at random from the list. In ChemicalEnvironments or'd decorators are split up into a base and list of sub-decorators. In our case we have two or'd decorators “\#6X3H2" and "\#7X2H1" which are split up into ('\#6', [' $\left.\mathrm{X} 3^{\prime},{ }^{\prime} \mathrm{H} 2{ }^{\prime}\right]$ ) and (' $\# 7^{\prime},\left[{ }^{\prime} \mathrm{X} 2^{\prime},{ }^{\prime} \mathrm{H} 1^{\prime}\right]$ ). There are six possible options for changing these decorators. In the most dramatic, all the or'd decorators are removed leaving us with the atom " $[* ;+0 ; \mathrm{A}]$." The simplest option is to remove just one sub-decorator (i.e. " $\mathrm{X} 3$ "). A complete list of these options is described in Table 3. When choosing an option, we only consider those that are possible with the current decorators. For example, if we had already removed all sub-decorators 


\begin{tabular}{|c|c|}
\hline Removal Category & Before change $\rightarrow$ After change \\
\hline Bond: only and'd & “] -, =; !@[" $\rightarrow$ “],$-=["$ \\
\hline Bond: all or'd & “]-,=;!@[" $\rightarrow$ “] $* ; ! @["$ \\
\hline \multirow{2}{*}{ Atom: one and'd } & "[\#6X3H2,\#7X2H1;+0;A:1]" $\rightarrow$ “[\#6X3H2,\#7X2H1;+0:1]" \\
\hline & “[\#6X3H2,\#7X2H1;+0; A : 1]" $\rightarrow$ “[\#6X3H2,\#7X2H1; A : 1]" \\
\hline Atom: all and'd & $“[\# 6 \times 3 \mathrm{H} 2, \# 7 \times 2 \mathrm{H} 1 ;+0 ; \mathrm{A}: 1] ” \rightarrow$ “[\#6X3H2,\#7X2H1:1]" \\
\hline Atom: all or'd & $"[\# 6 \times 3 \mathrm{H} 2, \# 7 \mathrm{X} 2 \mathrm{H} 1 ;+0 ; \mathrm{A}: 1] " \rightarrow "[* ;+0 ; \mathrm{A}: 1] "$ \\
\hline \multirow{2}{*}{ Atom: one or'd } & “[\#6X3H2,\#7X2H1;+0;A:1]" $\rightarrow$ “[\#7X2H1;+0;A:1]" \\
\hline & “[\#6X3H2,\#7X2H1;+0;A:1]" $\rightarrow$ “[\#6X3H2;+0;A:1]" \\
\hline Atom: all or'd bases & $"[\# 6 \mathrm{X} 3 \mathrm{H} 2, \# 7 \mathrm{X} 2 \mathrm{H} 1 ;+0 ; \mathrm{A}: 1] " \rightarrow$ "[*X4H2, *X3H1;+0;A : 1]" \\
\hline \multirow{2}{*}{$\begin{array}{l}\text { Atom: all sub-decorators from } \\
\text { one } \text { rrd }^{\prime}\end{array}$} & “[\#6X3H2,\#7X2H1;+0;A:1]" $\rightarrow$ “[\#6,\#7X2H1;+0;A:1]" \\
\hline & $"[\# 6 \times 3 \mathrm{H} 2, \# 7 \times 2 \mathrm{H} 1 ;+0 ; \mathrm{A}: 1] " \rightarrow$ "[\#6X3H2,\#7;+0;A:1]" \\
\hline \multirow{4}{*}{$\begin{array}{l}\text { Atom: one sub-decorator from } \\
\text { one } \text { rrd }^{\prime}\end{array}$} & “[\#6X3H2,\#7X2H1;+0;A:1]" $\rightarrow$ “[\#6H2,\#7X2H1;+0;A:1]" \\
\hline & $"[\# 6 \times 3 \mathrm{H} 2, \# 7 \mathrm{X} 2 \mathrm{H} 1 ;+0 ; \mathrm{A}: 1] " \rightarrow$ "[\#6X3,\#7X2H1;+0;A:1]" \\
\hline & $"[\# 6 \times 3 \mathrm{H} 2, \# 7 \mathrm{X} 2 \mathrm{H} 1 ;+0 ; \mathrm{A}: 1] " \rightarrow$ "[\#6X3H2,\#7H1;+0;A:1]" \\
\hline & $"[\# 6 \mathrm{X} 3 \mathrm{H} 2, \# 7 \mathrm{X} 2 \mathrm{H} 1 ;+0 ; \mathrm{A}: 1] " \rightarrow$ “[\#6X3H2,\#7X2;+0;A : 1]" \\
\hline \multirow{2}{*}{$\begin{array}{l}\text { Atom: all of one sub-decorator } \\
\text { category }\end{array}$} & “[\#6X3H2,\#7X2H1;+0; A:1]" $\rightarrow$ "[\#6H2,\#7H1;+0;A:1]" \\
\hline & 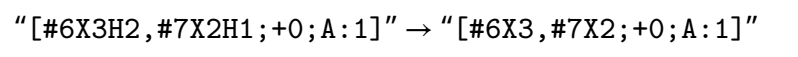 \\
\hline
\end{tabular}

Table 3. Categories for removing decorators as used in the Reducer. We will consider the SMIRKS "[\#6X3H 2 ,\#7X2H1;+0;A:1]-,=;! @ [\#1:2]," and provide all possible changes to atom : 1 or the bond.

and now had the atom " $[\# 6, \# 7 ;+0 ; \mathrm{A}]$, " then categories referencing removing sub-decorators would not be considered on the next round for this atom. Otherwise, the category is chosen randomly with equal probability for each.

\section{Validation and Discussion}

Now that we have initial implementations of the algorithms described above, we validate their behavior in two initial tests.

\subsection{Initial validation used the simple AlkEthOH molecule set}

The first check was to make sure ChemPer could generate SMIRKS on a very simplistic set of compounds. Specifically, we show that the SMIRKS we generate with ChemPer duplicate the typing of the AlkEthOH molecule set with smirnoff99Frosst version 1.0.7. AlkEthOH is a simple set of 42 molecules with only alkanes, ethers, and alcohols which was also used when testing SMARTY and SMIRKY. AlkEthOH has 5 bond, 7 angle, 16 proper torsion, and 9 nonbonded fragment types. As a side note, smirnoff99Frosst also includes improper torsion parameters, but AlkEthOH does not use these parameters as it only has single bonds between carbon, oxygen, and hydrogen. Fragments were clustered by which smirnoff99Frosst parameter they would be assigned. Then, these clusters were fed into SMIRKSifier to generate SMIRKS with all possible decorators based on this set of molecules (Section 3.4). In this test, the order of clusters were kept in the same order as their source parameters from smirnoff99Frosst.

The Reducer generalizes SMIRKS patterns by randomly attempting to remove decorators. The goal of this approach is to isolate which decorators are actually important for separating these clusters. However, the stochastic nature of this process means that rerunning the Reducer will result in different final SMIRKS patterns. We reduced the SMIRKS generated with SMIRKSifier 10 times using 1,000 iterations to explore the differences between reductions. Table 4 shows two examples of reduced SMIRKS patterns for the 


\begin{tabular}{lll}
\hline Label & Original & Reductions \\
\hline a1 & {$[*: 1] \sim[\# 6 \mathrm{XX}: 2]-[*: 3]$} & {$[*: 1] \sim[*: 2] \sim[*: 3]$} \\
& & {$[*: 1] \sim[*: 2] \sim[*: 3]$} \\
\hline a2 & {$[\# 1: 1]-[\# 6 \mathrm{X} 4: 2]-[\# 1: 3]$} & {$[*: 1] \sim ; ! @[* ; \mathrm{H} 2: 2] \sim ; ! @[*: 3]$} \\
& & {$[\# 1: 1] \sim[*: 2] \sim[\# 1: 3]$} \\
\hline a7 & {$[\# 6 r 4: 1]-; @[\# 6 r 4: 2]-; @[\# 6 r 4: 3]$} & {$[\# 6: 1] \sim[*: 2] \sim[* ; r 4 ; \mathrm{X} 4: 3]$} \\
& & {$[\# 6: 1] \sim[* ; r 4: 2] \sim[\# 6: 3]$} \\
\hline a8 & {$[! \# 1: 1]-[\# 6 r 4: 2]-; ! @[! \# 1: 3]$} & {$[*: 1] \sim[\# 6 r 4: 2] \sim[\# 8 ! r: 3]$} \\
& & {$[*: 1] \sim[\# 6 \mathrm{H} 1 \mathrm{r} 4: 2] \sim ; ! @[*: 3]$} \\
\hline a9 & {$[! \# 1: 1]-[\# 6 r 4: 2]-; ! @[\# 1: 3]$} & {$[\# 6, \# 8: 1] \sim[* ; r 4: 2] \sim[\# 1: 3]$} \\
& & {$[* \mathrm{X} 4, * \mathrm{X} 2: 1] \sim[* ; r 4: 2] \sim[\# 1: 3]$} \\
\hline a14 & {$[* ; r 6: 1] \sim ; @[* ; r 5: 2] \sim ; @[* ; r 5 ; \mathrm{x} 2: 3]$} & {$[*: 1] \sim ; @[* ; r 5: 2] \sim[* ; ! r: 3]$} \\
& & {$[*: 1] \sim ; @[* ; r 5: 2] \sim[* ; \mathrm{x} 0: 3]$} \\
\hline a27 & {$[*: 1]-[\# 8: 2]-[*: 3]$} & {$[*: 1] \sim[* ; \mathrm{X} 2: 2] \sim[*: 3]$} \\
& & {$[*: 1] \sim[\# 8: 2] \sim[*: 3]$} \\
\hline
\end{tabular}

Table 4. This table shows the SMIRKS patterns from smirnoff99Frosst (original). Then we show results from two different runs of Reducer. The stochastic decorator removal leads to different SMIRKS patterns for each reduction.

angle fragment types which retain different combinations of decorators. This highlights the complexity of generating SMIRKS since there are countless combinations of hierarchical lists which could match the same input clustering. The set of fully specified SMIRKS patterns and the 10 reduction iterations for each fragment type are available in the supporting information.

The stochastic nature of reduction affects the success of the final SMIRKS.

In most cases, the first SMIRKS pattern will have all of its decorators removed resulting in a fully generic pattern. For example, $[*: 1] \sim[*: 2] \sim[*: 3]$ will match any three atoms $(*)$ connected by any two bonds $(\sim)$, initially capturing any angle fragment. Due to the hierarchical nature of parameter assignment, this generic pattern will be matched to any fragment that doesn't match the patterns below. Some decorators consistently which remain in both reductions seem to capture the central chemistry. In parameters a7, a8, a9, and $\mathbf{a} 13$ the chemical feature which most affects the parameter is the size of the ring the angle is a part of. For both reductions, the ring size remains in the decorators on the central atoms. However, for other parameters the final decorators may not be the best combination. For example, in the first reduction for $\mathbf{a} 2$ the only decorators stored is the total hydrogen count on the central bond (H2) and than the bonds are not in a ring (!@). In this set of molecules, there is not much chemical diversity so this combination of decorators will match all of the necessary angles. However, in a more diverse set we can imagine wanting this angle to match hydrogen-carbon-hydrogen angles on a carbon with more than two hydrogens and we wouldn't want this same parameter to be applied to a non-carbon central atom. With this test, we had initial success in quickly generating SMIRKS to match the typing in smirnoff99Frosst, but there is still work to be done to optimize the Reducer.

\subsection{ChemPer was applied to produce protein SMIRKS}

One possible use of ChemPer would be to translate current atom typing force fields into the SMIRNOFF format. To this end, we attempted to generate SMRIKS patterns for the parameters in Amber99SB-ILDN for all fragment types. In this case we have all four valence types - bond, angle, proper torsion, and improper torsion - and the nonbonded types separated into Lennard-Jones and charge parameters. We generated and parameterized our protein with OpenMM, clustered fragments by parameter assigned, and used SMIRKSifier on those clusters. 


\subsubsection{A polypeptide was created with all possible amino acids.}

We needed a system with force field parameters which matched with a molecule in a cheminformatics toolkit in order to extract SMIRKS decorators. We started by creating a FASTA file, a text-based format to specify protein sequences, with 20 natural peptides capped at both ends by glycine. Then we used the OpenEye toolkits to parse the file, add hydrogens, and generate 3D coordinates for the polypeptide molecule. An open source tool, oeommtools, was used to convert the OpenEye molecule into an OpenMM system. The polypeptide was parameterized using the internal OpenMM force field file ('amber99sbildn.xml'). We generated clusters of fragments based on their assigned parameters (i.e. bonds were grouped by their equilibrium bond length and force constant). These clusters were then ready to be used with SMIRKSifier to generate SMIRKS patterns for each fragment type. We began by reducing the SMIRKS with 1,000 iterations. This resulted in significantly complex SMIRKS so we continued the reduction for a total of 5,000 iterations.

\subsubsection{A variety of ordering criteria was considered}

Choosing how to order fragment clusters can affect the way SMIRKS-based parameters are assigned. With the AlkEthOH test above, we kept clusters in the order of the parameters in smirnoff99Frosst. For the protein parameters however, there is not necessarily an obvious hierarchical order to the parameters. We tested four different ways of clustering SMIRKS patterns:

a) Longest SMIRKS: A SMIRKS pattern was generated with ClusterGraph for each cluster using only the indexed atoms. Then the clusters were sorted from longest to shortest SMIRKS pattern.

b) Shortest SMIRKS: The reverse of a), where the clusters are ordered from shortest to longest SMIRKS.

c) Largest Cluster: In this case, the clusters are used from largest to smallest based on the number of fragments they contain.

d) Smallest Cluster: The reverse of c), the clusters are sorted from smallest to largest based on the number of fragments.

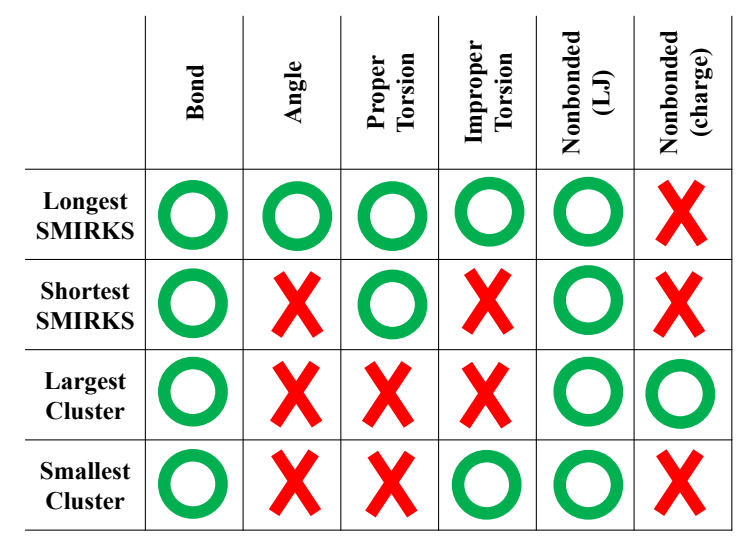

Figure 4. When creating SMIRKS based on AMBER protein force field parameters, we considered four ways of ordering the clustered fragments. First, we considered the decorators on the indexed atoms ordering by the Longest SMIRKS or the Shortest SMIRKS. Then, we considered the number of fragments checking the order by Largest Cluster or by Smallest Cluster. The order of clusters affected the success of ChemPer being able to capture this clustering in SMIRKS form. In this figure we show which ordering was successful for which fragment types.

We theorized we would have the most success with either a) or c), since then the more generic SMIRKS would match most of the fragments. Clusters which require more specialized chemistry would then be specified with more decoratorated SMIRKS patterns further down the hierarchy.

Success of the SMIRKSifier at maintaining the appropriate clustering varied by fragment type (Figure 4). We were able to make SMIRKS patterns which replicated force field parameter assignment for all fragment types. However, there was no ordering technique which was successful for all types of parameters. The Longest SMIRKS option was the best leading to successful SMIRKS patterns for all fragment types except the charge parameters. Charge and angle parameters appear to be the most difficult, given that only one ordering technique was successful. For charges, this may be due to the long distance effects on the parameter. Atoms many bonds away can affect the partial charge assigned to an atom. As discussed below (Section 5) there are still areas for further exploration when it comes to how non-tagged atoms are added to the ClusterGraphs.

The code used in this process is available to the public on GitHub at https://github.com/mobleylab/ chemper_proteins. The SMIRKS for each fragment type using each ordering technique are included in the 
supporting information.

\section{Future Work}

There are still questions to be explored for automatic SMIRKS generation for force field parameterization. We have shown some initial applications of ChemPer to generate SMIRKS patterns. There are use cases where ChemPer can be immediately useful in near future (Section 5.1). However, there are still aspects of the algorithms described above (Section 3) which require further optimization.

We need a better mechanism for determining the order of clustered fragments before feeding them into the SMIRKSifier.

The results for generating SMIRKS patterns for the polypeptide highlighted the importance of cluster ordering (Section 4.2.2). From these results we do not have an obvious answer about ordering clusters based on size. With further research it may be possible to establish a universal ordering based on already clustered fragments that could be considered. Perhaps we should instead consider the quantitative parameters assigned to the fragment and experiment with ordering parameters based on those instead. Another approach would be to consider hierarchical clustering algorithms [43], where the order of clusters could be determined before the stage of generating SMIRKS patterns.

We could improve the determination of where to add atoms and bonds to ClusterGraphs. In the ClusterGraph objects, decorators for multiple atoms and bonds are added to the corresponding nodes and edges. The placement of these atoms will affect the SMIRKS patterns which are created (Section 3.3). Currently, the decision about how to place atoms is made iteratively. The tagged and untagged atoms and bonds for the first molecule are added to the graph. Then the atoms and bonds for each subsequent molecule are added. First the tagged atoms are compared with those already stored in the graph, and reordered if necessary. Then, for each layer any neighboring atoms are considered and added to the node of the graph which most closely agrees with their decorators. This process is slow because it requires comparing atoms and bonds in each layer for each molecule added. It is also order dependent. That is if the molecules were added in a different order, it is possible atoms could be added to different nodes. A potentially better approach would be to compare the complete graph for each fragment before changing the placement of atoms. We could imagine building a SingleGraph for each fragment. Then, all of those graphs could be used simultaneously to determine whole to best order of atoms in each fragment. There are many algorithms for overlaying molecules which could be potentially adapted for this purposes, but they would need to be restrained to keep the tagged atoms aligned correctly for the given parameter. For example, angle parameters with atoms in the order 1,2,3 or 3,2,1 are equivalent for the purpose of assigning parameters, but should not be reordered to $2,1,3$ or 2,3,1. We should be able to learn from molecule overlay and graph similarity matching algorithms when improving this part of ChemPer.

\section{Decorator removal needs further examination.}

As highlighted in the AlkEthOH example above, the stochastic removal of decorators could cause potential problems when trying to make generally applicable SMIRKS patterns. A first step to improve this over training would be to split clusters into training and test groups. The Reducer could be used multiple times on the training set, but only the final sets of SMIRKS which also kept appropriate clustering in the test set would be considered for further use. An approach that would require an overhaul of the Reducer code would be to take a more deterministic approach where decorators are compared between the levels of the hierarchy. This would be simple especially for the and'd decorators, if the same are used in multiple levels at the top of the hierarchy then they are not helping to distinguish those levels. A deterministic approach for the or'd decorators would require comparing multiple sets of decorators and is less obvious. We could also consider untagged atoms more carefully. If they are the same for multiple clusters then they can be removed. When ChemPer adds a layer it adds all atoms in that layer, however we may only require one of those atoms to identify the important chemistry in a cluster of fragments. For example, consider the hydrogen Lennard-Jones parameters which are affected by the number of electron withdrawing groups attached to 
their carbon atom. When we want the cluster with only one electron withdrawing group, the other untagged atoms attached to the carbon are not necessary for separating this group from the others. The untagged atoms not representing the electron withdrawing group can be removed. We should be able to automatically detect that these are not the distinguishing features between clusters.

\subsection{Potential Applications}

One of the biggest benefits of the SMIRNOFF format is the ability to add new parameters without needing to add new chemical perception to any other parameter type. For example, in traditional force fields, when a new torsion is required, new atom type(s) must be created and then added to all other sections of the force field. However, in SMIRNOFF if a new parameter is needed, all one needs is the SMIRKS for that parameter and to add it to that section of the force field. While there is still science to be explored for the SMIRKS generation required for a general force field, there are smaller problems relevant to force field fitting where ChemPer could be more immediately useful.

SMIRKS could store bespoke parameters.

When general force field parameters are inaccurate for a particular molecule, bespoke parameters can be fit specifically for the molecule in question $[2,23]$. Recently introduced bespoke force field fitting tools allow parameters for both small molecules [25] and proteins [3] to be fit from quantum mechanical calculations before running simulations [10]. The energy barrier for bond rotation can be affected by the chemical environment many bonds away, making bespoke torsion fitting in combined with parameters from general force fields a common practice [40]. In any of these scenarios, it would be useful to be able to store and reuse these parameters again. ChemPer's SingleGraph can be used to create SMIRKS patterns where the whole molecule is specified with the atoms tagged for the relevant parameter. These parameters and SMIRKS could be added to the end of a SMIRNOFF file. Since parameters are assigned hierarchically and the bespoke SMIRKS would match only the specific molecule this SMIRNOFF could be used to parameterize the relevant molecule without affecting parameters assigned to other molecules in the system.

\section{ChemPer could help add chemical diversity to smirnoff99Frosst.}

There are elements, such as boron, which are common in organic chemistry, but have not been included in general force field parameterization. We could imagine fitting bespoke parameters for a variety of molecules containing these elements. ForceBalance is already available to automatically fit parameters based on reference quantum mechanical or experimental data [38]. Fragments could then be clustered by parameters that are within some threshold of each other. ChemPer's SMIRKSifier can be used to generate the SMIRKS for those clusters providing complete parameters and chemical perception to add to a SMIRNOFF.

\section{Conclusions}

ChemPer has proven to be capable of automatically generating relatively simple SMIRKS patterns which identify chemical differences between clustered fragments. It is successful at extracting and combining decorators for multiple fragments into one pattern. Each step in SMIRKS generation is separated into its own module (Section 3). We have shown some early success here by replicating smirnoff99Frosst parameters for AlkEthOH. We also automatically created SMIRKS patterns which replicated the typing in a common protein force field. The scripts required for both of these tests are available in the Supporting Information.

ChemPer is more efficient than SMIRKY. SMIRKY used a Monte Carlo algorithm starting with a single generic SMIRKS pattern, and the search resulted in a combinatorial explosion and associated sampling problems. As discussed in Section 2, there are many advantages to taking a more deterministic approach in ChemPer. In addition, SMIRKY required upwards of 100,000 iterations to be useful. ChemPer can create the high specific SMIRKS immediately and required around 5,000 iterations for generalization using the Reducer. This more efficient algorithm will make it a more practical combination with other force field parameterization tools.

While we showed some initial success, there are still areas for further experimentation with ChemPer. There are outstanding questions within ClusterGraph for how to overlay fragments and in Reducer for the 
best mechanism for making SMIRKS more general. Additionally, we need a mechanism for ordering clusters before feeding them into SMIRKSifier.

The development of ChemPer is in partnership with the Open Force Field Initiative, working to automate force field parameterization. Its development was an important step in removing the necessity for an expert to spend countless human hours assigning a force field's chemical perception. An improved ChemPer, addressing the issues detailed above, will be combined with tools from the Open Force Field Initiative to learn the quantitative force field parameters to automate the entire force field parameterization process. The combined machinery could change the future of force field science by allowing automated tools to optimize choices in reference data and functional form, therefore improving the results from simulations that depend on these models.

\section{Acknowledgements}

DLM appreciates financial support from the NIH grant 1R01GM108889-01. CCB appreciates financial support from The Molecular Sciences Software Institute under NSF grant ACI-1547580 which funded the development of ChemPer from the beginning. She thanks her MolSSI mentors, Daniel G. A. Smith and Jessica Nash, for their support and advice on good coding practices. The authors acknowledge many helpful discussions with the members of the Open Force Field Initiative, especially Victoria Lim and Jessica Maat for code review and feedback. We acknowledge Christopher Bayly for his advice about SMIRKS patterns and force field chemical perception. DLM serves on the scientific advisory board of OpenEye Scientific Software. The contents of this paper are solely the responsibility of the authors and do not necessarily represent the official views of any funding organization.

\section{Supporting Information}

The supporting information includes the extended results from validating ChemPer on AlkEthOH molecule set and the polypeptide. Specifically, we provide all ten of the repeated reduction of SMIRKS patterns for AlkEthOH and the SMIRKS for one protein example. The scripts and computer readable results for all of these tests are provided here and are available online at https://github.com/MobleyLab/chemper_SI.

\section{References}

[1] RDKit: Open Source Cheminformatics; 2018. doi.org/10.5281/zenodo.1222070.

[2] Ahmed S, Bidstrup SA, Kohl P, Ludovice P. Development of a new force field for polynorbornene. J Phys Chem B. 1998; 102(49):9783-9790.

[3] Allen A, Robertson MJ, Payne MC, Cole D. Development and Validation of the QUBE Protein Force Field. ChemRxiv. 2019 May; .

[4] Bannan CC, Mobley DL, ChemPer; 2018. doi.org/10.5281/zenodo.2548128.

[5] Baskin II, Winkler D, Tetko IV. A Renaissance of Neural Networks in Drug Discovery. Expert Opin Drug Discovery. 2016 Aug; 11(8):785-795.

[6] Cailliez F, Pernot P. Statistical Approaches to Forcefield Calibration and Prediction Uncertainty in Molecular Simulation. J Chem Phys. 2011 Feb; 134(5):054124.

[7] Case DA, Cheatham III TE, Darden T, Gohlke H, Luo R, Merz Jr KM, Onufriev A, Simmerling C, Wang B, Woods RJ. The Amber biomolecular simulation programs. J Comput Chem. 2005 Dec; 26(16):1668-1688.

[8] Chipot C, Pohorille A, editors. Free Energy Calculations: Theory and Applications in Chemistry and Biology. Springer; 2007.

[9] Christ CD, Mark AE, van Gunsteren WF. Feature Article Basic Ingredients of Free Energy Calculations: A Review. J Comput Chem. 2010 Jun; 31(8):1569-1582.

[10] Cole D, de Vaca IC, Jorgensen WL. Computation of Protein-Ligand Binding Free Energies using Quantum Mechanical Bespoke Force Fields. MedChemComm. 2019; . 
[11] Damm W, Frontera A, Tirado-Rives J, Jorgensen WL. OPLS All-Atom Force Field for Carbohydrates. J Comput Chem. 1997 Dec; 18(16):1955-1970.

[12] Daylight Chemical Information Systems Inc . Daylight Theory Manual. Daylight Chemical Information Systems Inc. 2011; Daylight Version 4.9.

[13] Deublein S, Metzler P, Vrabec J, Hasse H. Automated Development of Force Fields for the Calculation of Thermodynamic Properties: Acetonitrile as a Case Study. Mol Simul. 2013; 39(2):109-118.

[14] Ehrlich HC, Rarey M. Systematic Benchmark of Substructure Search in Molecular Graphs - From Ullmann to VF2. J Cheminform. 2012 Dec; 4(1):13.

[15] Fischer NM, van Maaren PJ, Ditz JC, Yildirim A, van der Spoel D. Properties of Organic Liquids When Simulated with Long-Range Lennard-Jones Interactions. J Chem Theory Comput. 2015 Jul; 11(7):2938-2944.

[16] Folgoc LL, Delingette H, Criminisi A, Ayache N. Quantifying Registration Uncertainty With Sparse Bayesian Modelling. IEEE Trans Med Imaging. 2017 Feb; 36(2):607-617.

[17] Geballe MT, Guthrie JP. The SAMPL3 blind prediction challenge: transfer energy overview. J Comput-Aided Mol Des. 2012 May; 26(5):489-496.

[18] Hagberg AA, Schult DA, Swart PJ. Exploring Network Structure, Dynamics, and Function Using NetworkX. In: Varoquaux G, Vaught T, Millman J, editors. Proceedings of the 7th Python in Science Conference Pasadena, CA USA; 2008. p. $11-15$.

[19] Hansen N, van Gunsteren WF. Practical Aspects of Free-Energy Calculations: A Review. J Chem Theory Comput. 2014 Jul; 10(7):2632-2647.

[20] Harder E, Damm W, Maple J, Wu C, Reboul M, Xiang JY, Wang L, Lupyan D, Dahlgren MK, Knight JL, Kaus JW, Cerutti DS, Krilov G, Jorgensen WL, Abel R, Friesner RA. OPLS3: A Force Field Providing Broad Coverage of Drug-like Small Molecules and Proteins. J Chem Theory Comput. 2016 Jan; 12(1):281-296.

[21] Hédin F, El Hage K, Meuwly M. A Toolkit to Fit Nonbonded Parameters from and for Condensed Phase Simulations. J Chem Inf Model. 2016 Aug; 56(8):1479-1489.

[22] Hess B, van der Vegt NFA. Hydration Thermodynamic Properties of Amino Acid Analogues: A Systematic Comparison of Biomolecular Force Fields and Water Models. J Phys Chem B. 2006 sep; 110(35):17616-17626.

[23] Holden D, Jelfs KE, Cooper Al, Trewin A, Willock DJ. Bespoke force field for simulating the molecular dynamics of porous organic cages. J Phys Chem C. 2012; 116(31):16639-16651.

[24] Hopkins CW, Roitberg AE. Fitting of Dihedral Terms in Classical Force Fields as an Analytic Linear Least-Squares Problem. J Chem Inf Model. 2014 Jul; 54(7):1978-1986.

[25] Horton J, Allen AE, Dodda LS, Cole DJ. QUBEKit: Automating the Derivation of Force Field Parameters from Quantum Mechanics. J Chem Inf Model. 2019; .

[26] Huang J, Rauscher S, Nawrocki G, Ran T, Feig M, de Groot BL, Grubmüller H, MacKerell Jr AD. CHARMM36m: An Improved Force Field for Folded and Intrinsically Disordered Proteins. Nat Methods. 2017 Jan; 14(1):71-73.

[27] Jiao D, Golubkov PA, Darden TA, Ren P. Calculation of Protein-ligand Binding Free Energy by Using a Polarizable Potential. PNAS. 2008 Apr; 105(17):6290-6295.

[28] Klepeis JL, Lindorff-Larsen K, Dror RO, Shaw DE. Long-Timescale Molecular Dynamics Simulations of Protein Structure and Function. Curr Opin Struct Biol. 2009 Apr; 19(2):120-127.

[29] Köster A, Spura T, Rutkai G, Kessler J, Wiebeler H, Vrabec J, Kühne TD. Assessing the Accuracy of Improved ForceMatched Water Models Derived from Ab Initio Molecular Dynamics Simulations. J Comput Chem. 2016 May; 37(19):1828-1838.

[30] Lesch V, Diddens D, Bernardes CES, Golub B, Dequidt A, Zeindlhofer V, Sega M, Schröder C. ForConX: A Forcefield Conversion Tool Based on XML. J Comput Chem. 2017 Apr; 38(9):629-638.

[31] Lindorff-Larsen K, Piana S, Palmo K, Maragakis P, Klepeis JL, Dror RO, Shaw DE. Improved side-chain torsion potentials for the Amber ff99SB protein force field. Proteins: Struct, Funct, Bioinf. 2010; 78(8):1950-1958.

[32] Marcou G, Rognan D. Optimizing Fragment and Scaffold Docking by Use of Molecular Interaction Fingerprints. J Chem Inf Model. 2006 Dec; 47(1):195-207. 
[33] Mishra SK, Calabró G, Loeffler HH, Michel J, Koča J. Evaluation of Selected Classical Force Fields for Alchemical Binding Free Energy Calculations of Protein-Carbohydrate Complexes. J Chem Theory Comput. 2015 Jul; 11(7):3333-3345.

[34] Mobley DL, Bannan CC, Rizzi A, Bayly Cl, Chodera JD, Lim VT, Lim NM, Beauchamp KA, Slochower DR, Shirts MR, et al. Escaping Atom Types in Force Fields Using Direct Chemical Perception. J Chem Theory Comput. 2018; 14(11):6076-6092.

[35] Mobley DL, Gilson MK. Predicting Binding Free Energies: Frontiers and Benchmarks. Annu Rev Biophys. 2017 May; 46:531-558.

[36] Mobley DL, Liu S, Cerutti DS, Swope WC, Rice JE. Alchemical prediction of hydration free energies for SAMPL. J Comput-Aided Mol Des. 2012 May; 26(5):551-562.

[37] OpenEye Scientific Software, Santa Fe, NM, OEChem Tooklkit; 2019. http://www.eyesopen.com.

[38] Pande V. Forcebalance: A Systematic, Reproducible, Statistically Driven Approach to More Accurate Molecular Dynamics Models. Biophys J. 2014 Jan; 106(2):44a.

[39] Pohorille A, Jarzynski C, Chipot C. Good Practices in Free-Energy Calculations. J Phys Chem B. 2010 Jan; 114(32):1023510253.

[40] Ponder JW, Case DA. Force fields for protein simulations. Adv Prot Chem. 2003; 66:27-85.

[41] Riniker S. Fixed-charge atomistic force fields for molecular dynamics simulations in the condensed phase: An overview. J Chem Inf Model. 2018; 58(3):565-578.

[42] Rizzi F, Najm H, Debusschere B, Sargsyan K, Salloum M, Adalsteinsson H, Knio O. Uncertainty Quantification in MD Simulations. Part II: Bayesian Inference of Force-Field Parameters. Multiscale Model Simul. 2012 Jan; 10(4):1460-1492.

[43] Rokach L, Maimon O. Clustering methods. In: Data mining and knowledge discovery handbook Springer; 2005.p. 321-352.

[44] Sellers BD, James NC, Gobbi A. A Comparison of Quantum and Molecular Mechanical Methods to Estimate Strain Energy in Druglike Fragments. J Chem Inf Model. 2017 Jun; 57(6):1265-1275.

[45] Shirts MR, Pitera JW, Swope WC, Pande VS. Extremely precise free energy calculations of amino acid side chain analogs: Comparison of common molecular mechanics force fields for proteins. J Chem Phys. 2003 Sep; 119(11):57405761.

[46] Shivakumar D, Deng Y, Roux B. Computations of Absolute Solvation Free Energies of Small Molecules Using Explicit and Implicit Solvent Model. J Chem Theory Comput. 2009 Apr; 5(4):919-930.

[47] Smith JS, Isayev O, Roitberg AE. ANI-1: An Extensible Neural Network Potential with DFT Accuracy at Force Field Computational Cost. Chem Sci. 2017; 8(4):3192-3203.

[48] Stahl M, Mauser H. Database Clustering with a Combination of Fingerprint and Maximum Common Substructure Methods. J Chem Inf Model. 2005 Mar; 45(3):542-548.

[49] Vanommeslaeghe K, MacKerell AD. Automation of the CHARMM General Force Field (CGenFF) I: Bond Perception and Atom Typing. J Chem Inf Model. 2012 Dec; 52:3144-3154.

[50] Wang J, Wolf RM, Caldwell JW, Kollman PA, Case DA. Development and testing of a general amber force field. J Comput Chem. 2004 Jul; 25(9):1157-1174.

[51] Xu P, Guidez EB, Bertoni C, Gordon MS. Perspective: Ab Initio Force Field Methods Derived from Quantum Mechanics. J Chem Phys. 2018 Mar; 148(9):090901.

[52] Zanette C, Bannan CC, Bayly Cl, Fass J, Gilson MK, Shirts MR, Chodera JD, Mobley DL. Toward learned chemical perception of force field typing rules. J Chem Theory Comput. 2018; 15(1):402-423. 


\section{Supporting Information - ChemPer: An Open Source tool for automatically generating SMIRKS patterns}

\section{Caitlin C. Bannan ${ }^{1}$ and David L. Mobley ${ }^{1,2}$}

${ }^{1}$ Department of Chemistry, University of California, Irvine CA 92697; ${ }^{2}$ Department of Pharmaceutical Science, University of California, Irvine CA 92697

*For correspondence: dmobley@mobleylab.org (DLM)

Included here are large tables of SMRIKS patterns generated with ChemPer and associated commentary. All scripts used to test ChemPer are provided in the electronic supporting information and are on GitHub at https://github.com/MobleyLab/chemper_Sl.

\section{SMIRKS patterns were generated to recreate smirnoff99Frosst typing in AlkEthOH}

In our first test, we parameterized the AlkEthOH molecule set with smirnoff99Frosst version 1.0.7. We clustered fragments by which parameter they would be assigned. Then, we used ChemPer's SMIRKSifier to generate a list of SMIRKS which maintains this clustering. Finally, the Reducer was used to remove decorators to make more general SMIRKS patterns. We ran the Reducer ten separate times. In the tables below, we report the final SMIRKS patterns for each parameter type: bonds (Table 1), angles (Table 2), proper torsions (Table 3), and nonbonded (Table 4). We use the symbol (x10) any time the exact same pattern was created in all ten Reducer runs as a way of shortening the table. In all cases, the first SMIRKS pattern was always reduced to be as generic as possible. For example, a bond would be $[*: 1][*: 2]$ matching any two atoms connected by any bond.

\begin{tabular}{|c|c|c|}
\hline \multicolumn{3}{|c|}{ Bond parameters in AlkEthOH } \\
\hline Label & Original & Reductions \\
\hline b1 & {$[\# 6 \times 4: 1]-[\# 6 \times 4: 2]$} & {$[*: 1] \sim[*: 2](\times 10)$} \\
\hline \multirow[t]{10}{*}{ b14 } & {$[\# 6: 1]-[\# 8: 2]$} & {$[*: 1] \sim ; ! @[*: 2]$} \\
\hline & & {$[*: 1] \sim ; ! @[*: 2]$} \\
\hline & & {$[*: 1] \sim ; ! @[*: 2]$} \\
\hline & & {$[*: 1] \sim[\# 8: 2]$} \\
\hline & & {$[*: 1] \sim[\# 8: 2]$} \\
\hline & & {$[*: 1] \sim ; ! @[*: 2]$} \\
\hline & & {$[*: 1] \sim ; ! @[*: 2]$} \\
\hline & & {$[*: 1] \sim ; ! @[*: 2]$} \\
\hline & & {$[*: 1] \sim ; ! @[*: 2]$} \\
\hline & & {$[*: 1] \sim[\# 8: 2]$} \\
\hline \multirow[t]{4}{*}{ b15 } & {$[\# 6 \mathrm{X} 4: 1]-[\# 8 \mathrm{X} 2 \mathrm{H0}: 2]$} & $\begin{array}{l}{[*: 1] \sim[\# 8 r 4, \# 8 r 5, \# 8 r 6, \# 8 r 7,} \\
\# 8 r 8, \# 8 r 9: 2]\end{array}$ \\
\hline & & {$[*: 1] \sim[* ; \mathrm{HO}: 2]$} \\
\hline & & {$[*: 1] \sim[* ; \mathrm{X} 2 ; \mathrm{x} 2: 2]$} \\
\hline & & {$[*: 1] \sim[* ; \mathrm{HO}: 2]$} \\
\hline
\end{tabular}




\section{Bond parameters in AlkEthOH}

\begin{tabular}{|c|c|c|}
\hline Label & Original & Reductions \\
\hline & & {$[*: 1] \sim ; @[\# 8: 2]$} \\
\hline & & {$[*: 1] \sim[* ; \mathrm{H} 0: 2]$} \\
\hline & & $\begin{array}{l}{[*: 1] \sim[\# 8 r 4, \# 8 r 5, \# 8 r 6, \# 8 r 7,} \\
\# 8 r 8, \# 8 r 9: 2]\end{array}$ \\
\hline & & {$[*: 1] \sim ; @[\# 8: 2]$} \\
\hline & & {$[*: 1] \sim ; @[* ; \mathrm{X} 2: 2]$} \\
\hline & & {$[*: 1] \sim ; @[\# 8: 2]$} \\
\hline b83 & {$[\# 6 \times 4: 1]-[\# 1: 2]$} & {$[*: 1] \sim[\# 1: 2](x 10)$} \\
\hline b87 & {$[\# 8: 1]-[\# 1: 2]$} & {$[\# 8: 1] \sim[\# 1: 2](\times 10)$} \\
\hline
\end{tabular}

Table 1. This table shows the SMIRKS patterns for bond parameters from smirnoff9gFrosst (original). Then we show results from ten different runs of Reducer. The stochastic decorator removal leads to different SMIRKS patterns for each reduction.

\begin{tabular}{|c|c|c|}
\hline \multicolumn{3}{|c|}{ Angle parameters in AlkEthOH } \\
\hline Label & Original & Reductions \\
\hline a1 & {$[*: 1] \sim[\# 6 \mathrm{X} 4: 2]-[*: 3]$} & {$[*: 1] \sim[*: 2] \sim[*: 3](x 10)$} \\
\hline \multirow[t]{10}{*}{ a2 } & {$[\# 1: 1]-[\# 6 \mathrm{X} 4: 2]-[\# 1: 3]$} & {$[*: 1] \sim ; ! @[* ; \mathrm{H} 2: 2] \sim ; ! @[*: 3]$} \\
\hline & & {$[\# 1: 1] \sim[*: 2] \sim[\# 1: 3]$} \\
\hline & & {$[\# 1: 1] \sim[*: 2] \sim[\# 1: 3]$} \\
\hline & & {$[*: 1] \sim ; ! @[* ; \mathrm{H} 2: 2] \sim ; ! @[*: 3]$} \\
\hline & & {$[*: 1] \sim ; ! @[* ; \mathrm{H} 2: 2] \sim ; ! @[*: 3]$} \\
\hline & & {$[*: 1] \sim ; ! @[* ; \mathrm{H} 2: 2] \sim ; ! @[*: 3]$} \\
\hline & & {$[*: 1] \sim ; ! @[* ; \mathrm{H} 2: 2] \sim ; ! @[*: 3]$} \\
\hline & & {$[*: 1] \sim ; ! @[* ; \mathrm{H} 2: 2] \sim ; ! @[*: 3]$} \\
\hline & & {$[*: 1] \sim ; ! @[* ; \mathrm{H} 2: 2] \sim ; ! @[*: 3]$} \\
\hline & & {$[\# 1: 1] \sim[*: 2] \sim[\# 1: 3]$} \\
\hline \multirow[t]{10}{*}{ a7 } & {$[\# 6 r 4: 1]-; @[\# 6 r 4: 2]-; @[\# 6 r 4: 3]$} & {$[\# 6: 1] \sim[*: 2] \sim[* ; r 4 ; \mathrm{X} 4: 3]$} \\
\hline & & {$[\# 6: 1] \sim[* ; r 4: 2] \sim[\# 6: 3]$} \\
\hline & & {$[\# 6: 1] \sim[*: 2] \sim[\# 6 ; r 4: 3]$} \\
\hline & & {$[\# 6: 1] \sim[* ; r 4: 2] \sim[\# 6: 3]$} \\
\hline & & {$[\# 6: 1] \sim[*: 2] \sim[\# 6 ; r 4: 3]$} \\
\hline & & {$[\# 6: 1] \sim[* ; r 4: 2] \sim[\# 6: 3]$} \\
\hline & & {$[\# 6: 1] \sim[* ; r 4: 2] \sim[\# 6: 3]$} \\
\hline & & {$[\# 6: 1] \sim[*: 2] \sim[\# 6 ; r 4: 3]$} \\
\hline & & {$[* ; r 4 ; \mathrm{X} 4: 1] \sim[*: 2] \sim[\# 6: 3]$} \\
\hline & & {$[\# 6: 1] \sim[* ; r 4: 2] \sim[\# 6: 3]$} \\
\hline \multirow[t]{5}{*}{ a8 } & {$[! \# 1: 1]-[\# 6 r 4: 2]-; ! @[! \# 1: 3]$} & {$[*: 1] \sim[\# 6 r 4: 2] \sim[\# 8 ! r: 3]$} \\
\hline & & {$[*: 1] \sim[\# 6 \mathrm{H} 1 \mathrm{r} 4: 2] \sim ; ! @[*: 3]$} \\
\hline & & {$[* ; r 4: 1] \sim[*: 2] \sim ; ! @[*: 3]$} \\
\hline & & {$[* ; r 4: 1] \sim[*: 2] \sim[\# 8 \mathrm{H} 1: 3]$} \\
\hline & & {$[*: 1] \sim ; @[\# 6 r 4: 2] \sim ; ! @[*: 3]$} \\
\hline
\end{tabular}




\section{Angle parameters in AlkEthOH}

\begin{tabular}{|c|c|c|}
\hline Label & Original & Reductions \\
\hline & & {$[* ; r 4: 1] \sim[*: 2] \sim ; ! @[*: 3]$} \\
\hline & & {$[*: 1] \sim[\# 6 \mathrm{H} 1 \mathrm{r} 4: 2] \sim ; ! @[*: 3]$} \\
\hline & & {$[* ; \mathrm{x} 2: 1] \sim[\# 6 \mathrm{r} 4: 2] \sim ; ! @[*: 3]$} \\
\hline & & {$[*: 1] \sim ; @[\# 6 r 4: 2] \sim ; ! @[*: 3]$} \\
\hline & & {$[* ; r 4: 1] \sim[*: 2] \sim[\# 8 ! r: 3]$} \\
\hline \multirow[t]{10}{*}{ a9 } & {$[! \# 1: 1]-[\# 6 r 4: 2]-; ! @[\# 1: 3]$} & {$[\# 6, \# 8: 1] \sim[* ; r 4: 2] \sim[\# 1: 3]$} \\
\hline & & {$[* \mathrm{X} 4, * \mathrm{X} 2: 1] \sim[* ; \mathrm{r} 4: 2] \sim[\# 1: 3]$} \\
\hline & & {$[* \mathrm{H} 1, * \mathrm{r} 4: 1] \sim[* ; \mathrm{r} 4: 2] \sim[\# 1: 3]$} \\
\hline & & {$[\# 6, \# 8: 1] \sim[* ; r 4: 2] \sim[\# 1: 3]$} \\
\hline & & {$[* \mathrm{X} 4, * \mathrm{X} 2: 1] \sim[* ; \mathrm{r} 4: 2] \sim[\# 1: 3]$} \\
\hline & & {$[* \mathrm{X} 4, * \mathrm{X} 2: 1] \sim[* ; \mathrm{r} 4: 2] \sim[\# 1: 3]$} \\
\hline & & {$[\# 6, \# 8: 1] \sim[* ; r 4: 2] \sim[\# 1: 3]$} \\
\hline & & {$[\# 6, \# 8: 1] \sim[* ; r 4: 2] \sim[\# 1: 3]$} \\
\hline & & {$[\# 6, \# 8: 1] \sim[* ; r 4: 2] \sim[\# 1: 3]$} \\
\hline & & {$[\# 6, \# 8: 1] \sim[* ; r 4: 2] \sim[\# 1: 3]$} \\
\hline \multirow[t]{10}{*}{ a14 } & {$[*: 1] \sim ; ! @[* ; r 5: 2] \sim ; @[* ; r 5: 3]$} & {$[*: 1] \sim ; @[* ; r 5: 2] \sim[* ; ! r: 3]$} \\
\hline & & {$[*: 1] \sim ; @[* ; r 5: 2] \sim[* ; \mathrm{x} 0: 3]$} \\
\hline & & {$[\# 6, \# 8 \mathrm{HO} 0: 1] \sim[* ; \mathrm{r} 5: 2] \sim[* \mathrm{X} 1, * \mathrm{X} 2 \mathrm{H} 1: 3]$} \\
\hline & & {$[*: 1] \sim ; @[* ; r 5: 2] \sim ; ! @[*: 3]$} \\
\hline & & {$[\# 6, \# 8 \mathrm{HO}: 1] \sim[* ; r 5: 2] \sim[* ; ! \mathrm{r}: 3]$} \\
\hline & & {$[*: 1] \sim ; @[* ; r 5: 2] \sim[* ; \mathrm{x} 0: 3]$} \\
\hline & & {$[* ; r 5: 1] \sim[*: 2] \sim ; ! @[*, *: 3]$} \\
\hline & & {$[* ; r 5: 1] \sim[*: 2] \sim ; ! @[*: 3]$} \\
\hline & & {$[* ; r 5: 1] \sim[*: 2] \sim[* ; \mathrm{x} 0: 3]$} \\
\hline & & {$[*: 1] \sim ; @[* ; r 5: 2] \sim ; ! @[*: 3]$} \\
\hline \multirow[t]{10}{*}{ a27 } & {$[*: 1]-[\# 8: 2]-[*: 3]$} & {$[*: 1] \sim[* ; \mathrm{X} 2: 2] \sim[*: 3]$} \\
\hline & & {$[*: 1] \sim[\# 8: 2] \sim[*: 3]$} \\
\hline & & {$[*: 1] \sim[\# 8: 2] \sim[*: 3]$} \\
\hline & & {$[*: 1] \sim[\# 8: 2] \sim[*: 3]$} \\
\hline & & {$[*: 1] \sim[\# 8: 2] \sim[*: 3]$} \\
\hline & & {$[*: 1] \sim[\# 8: 2] \sim[*: 3]$} \\
\hline & & {$[*: 1] \sim[\# 8: 2] \sim[*: 3]$} \\
\hline & & {$[*: 1] \sim[\# 8: 2] \sim[*: 3]$} \\
\hline & & {$[*: 1] \sim[\# 8: 2] \sim[*: 3]$} \\
\hline & & {$[*: 1] \sim[\# 8: 2] \sim[*: 3]$} \\
\hline
\end{tabular}

Table 2. This table shows the SMIRKS patterns for angle parameters from smirnoff99Frosst (original). Then we show results from ten different runs of Reducer. The stochastic decorator removal leads to different SMIRKS patterns for each reduction. 
Preprint ahead of submission - June 21, 2019

\section{Proper Torsion parameters in AlkEthOH}

\begin{tabular}{|c|c|c|}
\hline Label & Original & Reductions \\
\hline t1 & {$[*: 1]-[\# 6 \times 4: 2]-[\# 6 \times 4: 3]-[*: 4]$} & {$[*: 1] \sim[*: 2] \sim[*: 3] \sim[*: 4](\times 10)$} \\
\hline \multirow[t]{10}{*}{ t2 } & {$[\# 6 \times 4: 1]-[\# 6 \times 4: 2]-[\# 6 \times 4: 3]-[\# 6 \times 4: 4]$} & {$[\# 6: 1] \sim[*: 2] \sim[*: 3] \sim[* ; X 4: 4]$} \\
\hline & & {$[\# 6: 1] \sim[*: 2] \sim[*: 3] \sim[* ; \mathrm{X} 4: 4]$} \\
\hline & & {$[\# 6: 1] \sim[*: 2] \sim[*: 3] \sim[\# 6: 4]$} \\
\hline & & {$[\# 6: 1] \sim[*: 2] \sim[*: 3] \sim[\# 6: 4]$} \\
\hline & & {$[\# 6: 1] \sim[*: 2] \sim[*: 3] \sim[* ; \mathrm{X} 4: 4]$} \\
\hline & & {$[\# 6: 1] \sim[*: 2] \sim[*: 3] \sim[\# 6: 4]$} \\
\hline & & {$[* ; \mathrm{X} 4: 1] \sim[*: 2] \sim[*: 3] \sim[\# 6: 4]$} \\
\hline & & {$[\# 6: 1] \sim[*: 2] \sim[*: 3] \sim[\# 6: 4]$} \\
\hline & & {$[\# 6: 1] \sim[*: 2] \sim[*: 3] \sim[\# 6: 4]$} \\
\hline & & {$[\# 6: 1] \sim[*: 2] \sim[*: 3] \sim[* ; \mathrm{X} 4: 4]$} \\
\hline \multirow[t]{10}{*}{ t3 } & {$[\# 1: 1]-[\# 6 \times 4: 2]-[\# 6 \times 4: 3]-[\# 1: 4]$} & {$[*: 1] \sim ; ! @[*: 2] \sim[*: 3] \sim ; ! @[*: 4]$} \\
\hline & & {$[*: 1] \sim ; ! @[*: 2] \sim[*: 3] \sim ; ! @[*: 4]$} \\
\hline & & {$[\# 1: 1] \sim[*: 2] \sim[*: 3] \sim[*: 4]$} \\
\hline & & {$[*: 1] \sim[*: 2] \sim[*: 3] \sim[\# 1: 4]$} \\
\hline & & {$[*: 1] \sim[*: 2] \sim[*: 3] \sim[\# 1: 4]$} \\
\hline & & {$[\# 1: 1] \sim[*: 2] \sim[*: 3] \sim[*: 4]$} \\
\hline & & {$[*: 1] \sim ; ! @[*: 2] \sim[*: 3] \sim ; ! @[*: 4]$} \\
\hline & & {$[*: 1] \sim[*: 2] \sim[*: 3] \sim[\# 1: 4]$} \\
\hline & & {$[*: 1] \sim ; ! @[*: 2] \sim[*: 3] \sim ; ! @[*: 4]$} \\
\hline & & {$[*: 1] \sim[*: 2] \sim[*: 3] \sim[\# 1: 4]$} \\
\hline \multirow[t]{10}{*}{ t4 } & {$[\# 1: 1]-[\# 6 \times 4: 2]-[\# 6 \times 4: 3]-[\# 6 \times 4: 4]$} & {$[*: 1] \sim ; @[*: 2] \sim[*: 3] \sim[\# 1: 4]$} \\
\hline & & {$[*: 1] \sim ; @[*: 2] \sim[*: 3] \sim[\# 1: 4]$} \\
\hline & & {$[*: 1] \sim ; @[*: 2] \sim[*: 3] \sim[\# 1: 4]$} \\
\hline & & {$[*: 1] \sim ; @[*: 2] \sim[*: 3] \sim[\# 1: 4]$} \\
\hline & & {$[*: 1] \sim ; @[*: 2] \sim[*: 3] \sim[\# 1: 4]$} \\
\hline & & {$[*: 1] \sim ; @[*: 2] \sim[*: 3] \sim[\# 1: 4]$} \\
\hline & & {$[\# 6: 1] \sim[*: 2] \sim[*: 3] \sim[\# 1: 4]$} \\
\hline & & {$[*: 1] \sim ; @[*: 2] \sim[*: 3] \sim[\# 1: 4]$} \\
\hline & & {$[*: 1] \sim ; @[*: 2] \sim[*: 3] \sim[\# 1: 4]$} \\
\hline & & {$[\# 6: 1] \sim[*: 2] \sim[*: 3] \sim[\# 1: 4]$} \\
\hline \multirow[t]{9}{*}{ t5 } & {$[\# 8 \times 2: 1]-[\# 6 \times 4: 2]-[\# 6 \times 4: 3]-[\# 8 \times 2: 4]$} & {$[\# 8: 1] \sim[*: 2] \sim[*: 3] \sim[\# 8: 4]$} \\
\hline & & {$[\# 8: 1] \sim[*: 2] \sim[*: 3] \sim[\# 8: 4]$} \\
\hline & & {$[* ; \mathrm{X} 2: 1] \sim[*: 2] \sim[*: 3] \sim[* ; \mathrm{X} 2: 4]$} \\
\hline & & {$[\# 8: 1] \sim[*: 2] \sim[*: 3] \sim[* ; \mathrm{X} 2: 4]$} \\
\hline & & {$[\# 8: 1] \sim[*: 2] \sim[*: 3] \sim[* ; \mathrm{X} 2: 4]$} \\
\hline & & {$[\# 8: 1] \sim[*: 2] \sim[*: 3] \sim[\# 8: 4]$} \\
\hline & & {$[* ; \mathrm{X} 2: 1] \sim[*: 2] \sim[*: 3] \sim[* ; \mathrm{X} 2: 4]$} \\
\hline & & {$[\# 8: 1] \sim[*: 2] \sim[*: 3] \sim[\# 8: 4]$} \\
\hline & & {$[\# 8: 1] \sim[*: 2] \sim[*: 3] \sim[\# 8: 4]$} \\
\hline
\end{tabular}


Preprint ahead of submission - June 21, 2019

Proper Torsion parameters in AlkEthOH

\begin{tabular}{|c|c|c|}
\hline Label & Original & Reductions \\
\hline & & {$[* ; \mathrm{X} 2: 1] \sim[*: 2] \sim[*: 3] \sim[* ; \mathrm{X} 2: 4]$} \\
\hline \multirow[t]{10}{*}{ t9 } & {$[\# 1: 1]-[\# 6 \times 4: 2]-[\# 6 \times 4: 3]-[\# 8 \times 2: 4]$} & {$[* ; \mathrm{X} 2: 1] \sim[*: 2] \sim[*: 3] \sim[\# 1: 4]$} \\
\hline & & {$[\# 8: 1] \sim[*: 2] \sim[*: 3] \sim[\# 1: 4]$} \\
\hline & & {$[\# 8: 1] \sim[*: 2] \sim[*: 3] \sim[\# 1: 4]$} \\
\hline & & {$[\# 8: 1] \sim[*: 2] \sim[*: 3] \sim[\# 1: 4]$} \\
\hline & & {$[* ; \mathrm{X} 2: 1] \sim[*: 2] \sim[*: 3] \sim[\# 1: 4]$} \\
\hline & & {$[* ; \mathrm{X} 2: 1] \sim[*: 2] \sim[*: 3] \sim[\# 1: 4]$} \\
\hline & & {$[\# 8: 1] \sim[*: 2] \sim[*: 3] \sim[\# 1: 4]$} \\
\hline & & {$[\# 8: 1] \sim[*: 2] \sim[*: 3] \sim[\# 1: 4]$} \\
\hline & & {$[\# 8: 1] \sim[*: 2] \sim[*: 3] \sim[\# 1: 4]$} \\
\hline & & {$[\# 8: 1] \sim[*: 2] \sim[*: 3] \sim[\# 1: 4]$} \\
\hline \multirow[t]{10}{*}{ t84 } & {$[*: 1]-[\# 6 \mathrm{X} 4: 2]-[\# 8 \mathrm{X} 2: 3]-[\# 1: 4]$} & {$[*: 1] \sim[*: 2] \sim[\# 8: 3] \sim[*: 4]$} \\
\hline & & {$[*: 1] \sim[*: 2] \sim[\# 8: 3] \sim[*: 4]$} \\
\hline & & {$[*: 1] \sim[*: 2] \sim ; ! @[*: 3] \sim[*: 4]$} \\
\hline & & {$[*: 1] \sim[*: 2] \sim ; ! @[*: 3] \sim[*: 4]$} \\
\hline & & {$[*: 1] \sim[*: 2] \sim ; ! @[*: 3] \sim[*: 4]$} \\
\hline & & {$[*: 1] \sim[*: 2] \sim ; ! @[*: 3] \sim[*: 4]$} \\
\hline & & {$[*: 1] \sim[*: 2] \sim ; ! @[*: 3] \sim[*: 4]$} \\
\hline & & {$[*: 1] \sim[*: 2] \sim ; ! @[*: 3] \sim[*: 4]$} \\
\hline & & {$[*: 1] \sim[*: 2] \sim[\# 8: 3] \sim[*: 4]$} \\
\hline & & {$[*: 1] \sim[*: 2] \sim ; ! @[*: 3] \sim[*: 4]$} \\
\hline \multirow[t]{10}{*}{ t85 } & {$[\# 6 \times 4: 1]-[\# 6 \mathrm{X} 4: 2]-[\# 8 \mathrm{X} 2 \mathrm{H} 1: 3]-[\# 1: 4]$} & {$[\# 6: 1] \sim[*: 2] \sim[\# 8: 3] \sim[*: 4]$} \\
\hline & & {$[\# 6: 1] \sim[*: 2] \sim ; ! @[*: 3] \sim[*: 4]$} \\
\hline & & {$[\# 6: 1] \sim[*: 2] \sim[\# 8: 3] \sim[*: 4]$} \\
\hline & & {$[* ; \mathrm{X} 4: 1] \sim[*: 2] \sim[\# 8: 3] \sim[*: 4]$} \\
\hline & & {$[\# 6: 1] \sim[*: 2] \sim ; ! @[*: 3] \sim[*: 4]$} \\
\hline & & {$[\# 6: 1] \sim[*: 2] \sim[\# 8: 3] \sim[*: 4]$} \\
\hline & & {$[\# 6: 1] \sim[*: 2] \sim[\# 8: 3] \sim[*: 4]$} \\
\hline & & {$[* ; \mathrm{X} 4: 1] \sim[*: 2] \sim ; ! @[*: 3] \sim[*: 4]$} \\
\hline & & {$[\# 6: 1] \sim[*: 2] \sim[\# 8: 3] \sim[*: 4]$} \\
\hline & & {$[\# 6: 1] \sim[*: 2] \sim[\# 8: 3] \sim[*: 4]$} \\
\hline \multirow[t]{9}{*}{ t86 } & {$[*: 1]-[\# 6 \mathrm{X} 4: 2]-[\# 8 \mathrm{X} 2 \mathrm{H} 0: 3]-[*: 4]$} & {$[*: 1] \sim[\# 8: 2] \sim ; @[*: 3] \sim[*: 4]$} \\
\hline & & {$[*: 1] \sim[\# 8: 2] \sim ; @[*: 3] \sim[*: 4]$} \\
\hline & & {$[*: 1] \sim ; @[\# 8: 2] \sim[*: 3] \sim[*: 4]$} \\
\hline & & {$[*: 1] \sim[\# 8: 2] \sim ; @[*: 3] \sim[*: 4]$} \\
\hline & & {$[*: 1] \sim[* ; \mathrm{HO}: 2] \sim[*: 3] \sim[*: 4]$} \\
\hline & & {$[*: 1] \sim ; @[\# 8: 2] \sim[*: 3] \sim[*: 4]$} \\
\hline & & {$[*: 1] \sim[\# 8: 2] \sim ; @[*: 3] \sim[*: 4]$} \\
\hline & & {$[*: 1] \sim[* ; \mathrm{HO}: 2] \sim[*: 3] \sim[*: 4]$} \\
\hline & & {$[*: 1] \sim ; @[\# 8: 2] \sim[*: 3] \sim[*: 4]$} \\
\hline
\end{tabular}


Preprint ahead of submission - June 21, 2019

Proper Torsion parameters in AlkEthOH

\begin{tabular}{|c|c|c|}
\hline Label & Original & Reductions \\
\hline & & {$[*: 1] \sim ; @[\# 8: 2] \sim[*: 3] \sim[*: 4]$} \\
\hline \multirow[t]{10}{*}{ t87 } & {$[\# 6 \times 4: 1]-[\# 6 \times 4: 2]-[\# 8 \times 2 \mathrm{HO}: 3]-[\# 6 \times 4: 4]$} & {$[*: 1] \sim ; @[*: 2] \sim ; @[\# 8: 3] \sim[*: 4]$} \\
\hline & & {$[*: 1] \sim ; @[*: 2] \sim[* ; \mathrm{HO}: 3] \sim[*: 4]$} \\
\hline & & {$[*: 1] \sim ; @[*: 2] \sim[\# 8: 3] \sim ; @[*: 4]$} \\
\hline & & {$[\# 6: 1] \sim[*: 2] \sim ; @[\# 8: 3] \sim[*: 4]$} \\
\hline & & {$[*: 1] \sim ; @[*: 2] \sim[* ; \mathrm{X} 2 ; \mathrm{X} 2: 3] \sim[*: 4]$} \\
\hline & & {$[*: 1] \sim ; @[*: 2] \sim ; @[\# 8: 3] \sim[*: 4]$} \\
\hline & & {$[*: 1] \sim ; @[*: 2] \sim[* ; \mathrm{HO}: 3] \sim[*: 4]$} \\
\hline & & {$[*: 1] \sim ; @[*: 2] \sim[\# 8: 3] \sim ; @[*: 4]$} \\
\hline & & {$[*: 1] \sim ; @[*: 2] \sim[* ; \mathrm{X} 2 ; \mathrm{X} 2: 3] \sim[*: 4]$} \\
\hline & & {$[*: 1] \sim ; @[*: 2] \sim ; @[\# 8: 3] \sim[*: 4]$} \\
\hline \multirow[t]{10}{*}{ t89 } & {$[\# 6 \times 4: 1]-[\# 8 \times 2: 2]-[\# 6 \times 4: 3]-[\# 8 \times 2: 4]$} & {$[*: 1] \sim[* ; \mathrm{HO}: 2] \sim[*: 3] \sim[\# 8: 4]$} \\
\hline & & {$[*: 1] \sim[\# 8: 2] \sim ; @[*: 3] \sim[* ; \mathrm{X} 2: 4]$} \\
\hline & & {$[*: 1] \sim[\# 8, \# 8, \# 8: 2] \sim ; @[*: 3] \sim[\# 8, \# 8: 4]$} \\
\hline & & {$[*: 1] \sim ; @[* ; \mathrm{X} 2: 2] \sim[*: 3] \sim[\# 8: 4]$} \\
\hline & & {$[*: 1] \sim ; @[\# 8: 2] \sim[*: 3] \sim[\# 8: 4]$} \\
\hline & & {$[*: 1] \sim ; @[\# 8: 2] \sim[*: 3] \sim[\# 8: 4]$} \\
\hline & & {$[*: 1] \sim[\# 8: 2] \sim ; @[*: 3] \sim[\# 8: 4]$} \\
\hline & & {$[*: 1] \sim[\# 8: 2] \sim ; @[*: 3] \sim[* ; \mathrm{X} 2: 4]$} \\
\hline & & {$[*: 1] \sim ; @[\# 8: 2] \sim[*: 3] \sim[* ; \mathrm{X} 2: 4]$} \\
\hline & & {$[*: 1] \sim[\# 8: 2] \sim ; @[*: 3] \sim[\# 8: 4]$} \\
\hline
\end{tabular}

Table 3. This table shows the SMIRKS patterns for proper torsion parameters from smirnoff99Frosst (original). Then we show results from ten different runs of Reducer. The stochastic decorator removal leads to different SMIRKS patterns for each reduction.

\begin{tabular}{lll}
\hline & \multicolumn{2}{c}{ Nonbond parameters in AlkEthOH } \\
\hline Label & Original & Reductions \\
\hline $\mathbf{n} \mathbf{n 3}$ & {$[\# 1: 1]-[\# 6 \mathrm{X} 4]$} & {$[*: 1](\mathrm{X} 10)$} \\
& {$[\# 1: 1]-[\# 6 \mathrm{X} 4]-[\# 7, \# 8, \# 9, \# 16, \# 17, \# 35]$} & {$[*: 1] \sim[*] \sim[\# 8]$} \\
& {$[*: 1] \sim[*] \sim[* ; \mathrm{X} 2]$} \\
& {$[*: 1] \sim[*] \sim[* ; \mathrm{X} 2]$} \\
& {$[*: 1] \sim[*] \sim[\# 8]$} \\
& {$[*: 1] \sim[*] \sim[\# 8]$} \\
& {$[*: 1] \sim[*] \sim[\# 8]$} \\
& {$[*: 1] \sim[*] \sim[\# 8]$} \\
& {$[*: 1] \sim[*] \sim[\# 8]$} \\
& & {$[*: 1] \sim[*] \sim[\# 8]$} \\
& {$[\# 1: 1]-[\# 6 \mathrm{X} 4](-[\# 7, \# 8, \# 9, \# 16, \# 17, \quad[*: 1] \sim[*] \sim[\# 8]$} \\
\hline n4 & {$[*: 1] \sim[*](\sim[\# 8]) \sim[\# 8]$} \\
& & {$[*: 1] \sim[*](\sim[\# 8]) \sim[* ; \mathrm{X} 2]$}
\end{tabular}


Preprint ahead of submission - June 21, 2019

Nonbond parameters in AlkEthOH

\begin{tabular}{|c|c|c|}
\hline Label & Original & Reductions \\
\hline & & {$[*: 1] \sim[*](\sim[* ; \mathrm{X} 2]) \sim[* ; \mathrm{X} 2]$} \\
\hline & & {$[*: 1] \sim[*](\sim[\# 8]) \sim[* ; \mathrm{X} 2]$} \\
\hline & & {$[*: 1] \sim[*](\sim[\# 8]) \sim[\# 8]$} \\
\hline & & {$[*: 1] \sim[*](\sim[\# 8]) \sim[\# 8]$} \\
\hline & & {$[*: 1] \sim[*](\sim[\# 8]) \sim[\# 8]$} \\
\hline & & {$[*: 1] \sim[*](\sim[\# 8]) \sim[\# 8]$} \\
\hline & & {$[*: 1] \sim[*](\sim[* ; \mathrm{HO} ; \mathrm{x} 2]) \sim[* ; \mathrm{X} 2]$} \\
\hline & & {$[*: 1] \sim[*](\sim[\# 8]) \sim[\# 8]$} \\
\hline \multirow[t]{10}{*}{ n5 } & $\begin{array}{l}{[\# 1: 1]-[\# 6 \times 4](-[\# 7, \# 8, \# 9, \# 16, \# 17,} \\
\# 35])(-[\# 7, \# 8, \# 9, \# 16, \# 17, \# 35])- \\
{[\# 7, \# 8, \# 9, \# 16, \# 17, \# 35]}\end{array}$ & {$[*: 1] \sim[*](\sim[\# 8])(\sim[\# 8]) \sim[* ; \mathrm{X} 2]$} \\
\hline & & $\begin{array}{l}{[*: 1] \sim[* ; \mathrm{H} 1](\sim ; @[\# 8]) \sim[\# 8 \mathrm{r} 5,} \\
\# 8 \mathrm{r} 7, \# 8 \mathrm{r} 9]\end{array}$ \\
\hline & & $\begin{array}{l}{[*: 1] \sim[* ; \mathrm{H} 1](\sim ; @[\# 8]) \sim[\# 8 r 5,} \\
\# 8 r 7, \# 8 r 9]\end{array}$ \\
\hline & & {$[*: 1] \sim[* ; \mathrm{H} 1](\sim ; @[\# 8]) \sim ; @[\# 8]$} \\
\hline & & {$[*: 1] \sim[*](\sim[\# 8])(\sim[\# 8]) \sim[\# 8]$} \\
\hline & & {$[*: 1] \sim[*](\sim[\# 8])(\sim[\# 8]) \sim[\# 8]$} \\
\hline & & {$[*: 1] \sim[* ; \mathrm{H} 1](\sim ; @[\# 8]) \sim[* ; \mathrm{HO}]$} \\
\hline & & {$[*: 1] \sim[* ; H 1](\sim ; @[\# 8]) \sim ; @[\# 8]$} \\
\hline & & {$[*: 1] \sim[*](\sim[\# 8])(\sim[\# 8]) \sim[* ; \mathrm{X} 2]$} \\
\hline & & {$[*: 1] \sim[*](\sim[\# 8])(\sim[\# 8]) \sim[* ; \mathrm{X} 2]$} \\
\hline \multirow[t]{10}{*}{ n12 } & {$[\# 1: 1]-[\# 8]$} & {$[*: 1] \sim[*] \sim ; ! @[\# 6]$} \\
\hline & & {$[*: 1] \sim[\# 8]$} \\
\hline & & {$[*: 1] \sim[*] \sim ; ! @[\# 6]$} \\
\hline & & {$[*: 1] \sim[\# 8]$} \\
\hline & & {$[*: 1] \sim[*] \sim ; ! @[\# 6]$} \\
\hline & & {$[*: 1] \sim[\# 8]$} \\
\hline & & {$[*: 1] \sim[\# 8]$} \\
\hline & & {$[*: 1] \sim[*] \sim ; ! @[\# 6]$} \\
\hline & & {$[*: 1] \sim[\# 8]$} \\
\hline & & {$[*: 1] \sim[\# 8]$} \\
\hline \multirow[t]{9}{*}{ n16 } & {$[\# 6 \times 4: 1]$} & {$[*: 1] \sim[\# 1]$} \\
\hline & & {$[*: 1] \sim[\# 1]$} \\
\hline & & {$[\# 6: 1]$} \\
\hline & & {$[\# 6: 1]$} \\
\hline & & {$[*: 1](\sim[*]) \sim[*]$} \\
\hline & & {$[*: 1](\sim[*]) \sim[*]$} \\
\hline & & {$[* ; \times 2: 1]$} \\
\hline & & {$[*: 1] \sim[\# 1]$} \\
\hline & & {$[\# 6: 1]$} \\
\hline
\end{tabular}




\begin{tabular}{|c|c|c|}
\hline \multicolumn{3}{|c|}{ Nonbond parameters in AlkEthOH } \\
\hline Label & Original & Reductions \\
\hline & & {$[\# 6: 1]$} \\
\hline \multirow[t]{10}{*}{ n18 } & {$[\# 8 \mathrm{X} 2 \mathrm{H} 0+0: 1]$} & {$[* ; \mathrm{HO} ; \mathrm{x} 2: 1]$} \\
\hline & & {$[\# 8: 1]$} \\
\hline & & {$[\# 8: 1]$} \\
\hline & & {$[* ; \mathrm{HO} ; \mathrm{x} 2: 1]$} \\
\hline & & {$[\# 8: 1]$} \\
\hline & & {$[* ; \mathrm{X} 2: 1]$} \\
\hline & & {$[\# 8: 1]$} \\
\hline & & {$[* ; \mathrm{HO} ; \mathrm{x} 2: 1]$} \\
\hline & & {$[* ; X 2: 1]$} \\
\hline & & {$[\# 8: 1]$} \\
\hline \multirow[t]{10}{*}{ n19 } & {$[\# 8 \times 2 H 1+0: 1]$} & {$[\# 8: 1] \sim ; ! @[*]$} \\
\hline & & {$[\# 8 \times 0: 1]$} \\
\hline & & {$[*: 1] \sim ; ! @[* ; \mathrm{H} 1 ; \mathrm{x} 2] \sim[\# 1]$} \\
\hline & & {$[\# 8: 1] \sim ; ! @[*]$} \\
\hline & & {$[*: 1](\sim ; ! @[\# 6]) \sim[*]$} \\
\hline & & {$[\# 8 \mathrm{H} 1: 1]$} \\
\hline & & {$[*: 1] \sim ; ! @[* ; \mathrm{H} 1 ; \mathrm{x} 2] \sim[\# 1]$} \\
\hline & & {$[\# 8 \mathrm{H} 1: 1]$} \\
\hline & & {$[\# 8 ! r: 1]$} \\
\hline & & {$[*: 1](\sim ; ! @[\# 6]) \sim[*]$} \\
\hline
\end{tabular}

Table 4. This table shows the SMIRKS patterns for nonbonded parameters from smirnoffg9Frosst (original). Then we show results from ten different runs of Reducer. The stochastic decorator removal leads to different SMIRKS patterns for each reduction.

\section{SMIRKS patterns were created to reproduce a protein force field}

We generated SMRIKS patterns for the parameters in Amber99SB-ILDN using a simple polypeptide. As described in the main text, we used OpenEye and OpenMM to parameterize a polypeptide containing all 20 natural amino acids capped at both ends by glycine. Fragments were clustered by their assigned force field parameter, then ChemPer was used to generate SMIRKS patterns with a total of 5,000 iterations in the Reducer. In these tests, we tried a variety of techniques for ordering clusters before creating the SMIRKS. The most successful ordering strategy was based on the length of the SMIRKS pattern where the clusters with the most variation of decorators were put at the top of the hierarchy. We share the SMIRKS created with this ordering technique for all valence all four valence types - bond (Table 5), angle (Table 6), proper torsion (Table 8), and improper torsion (Table 7) - and the Lennard-Jones (Table 9). In all tables, the SMIRKS are listed with the quantitative force field parameter assigned to its cluster.

For the polypeptide used in this test there were 199 partial charge clusters. However, the Reducer was run for the same number of iterations with charges as the other parameters. 5,000 iterations was not sufficient to reduce the SMIRKS patterns for such a large number of clusters. For this reason, we are not showing a table of SMIRKS for partial charges here. They are accessible in the electronic supporting information and online at https://github.com/MobleyLab/chemper_SI.

Improper torsion parameters may require more work before the SMIRKS could be used to assign parameters. In these tests, used only fragments that were assigned an improper torsion when creating the SMIRKS patterns. However, there are many trivalent atoms that are not assigned improper torsion parameters. That 
means the generalized SMIRKS patterns in Table 7 could match atoms which should not be assigned the corresponding parameter. To address this issue, you would need to add a cluster of all atoms which do not get an improper parameter as a separate cluster before running ChemPer.

As discussed in the main text, there is still room for improvement in ChemPer. The SMIRKS patterns created for the polypeptides highlight a couple of these areas. In many of these cases there is a significant difference in the number of decorators in different SMIRKS patterns. It is possible that some clusters will require more decorators to capture the required chemistry, however some of these are very extreme with most of the decorators remaining on nearly all atoms. This reinforces the idea that a slightly more deterministic approach to determining where to remove decorators would be preferable.

\begin{tabular}{|c|c|c|}
\hline \multicolumn{3}{|r|}{ Polypeptide bond parameters } \\
\hline$k$ & $l$ & SMIRKS \\
\hline 310 & 1.526 & {$[*: 1] \sim[*: 2]$} \\
\hline 434 & 1.010 & {$[*: 1] \sim[\# 1: 2]$} \\
\hline 340 & 1.090 & {$[\# 6: 1] \sim[\# 1: 2]$} \\
\hline 337 & 1.449 & {$[\# 7: 1] \sim[*: 2] \sim[*]$} \\
\hline 481 & 1.340 & {$[\# 7: 1](\sim[\# 1])(\sim[\# 6: 2]) \sim[* \mathrm{X} 1, * \mathrm{X} 4]$} \\
\hline 490 & 1.335 & {$[\# 6 \times 3: 1](\sim[\# 8]) \sim[*: 2]$} \\
\hline 317 & 1.522 & {$[* ; \mathrm{X} 4: 1] \sim[\# 6 \mathrm{X} 3: 2]$} \\
\hline 469 & 1.400 & {$[*: 1]:[*: 2]$} \\
\hline 656 & 1.250 & {$[*: 1] \sim[\# 8: 2]$} \\
\hline 227 & 1.810 & {$[*: 1] \sim[\# 16: 2]$} \\
\hline 367 & 1.080 & 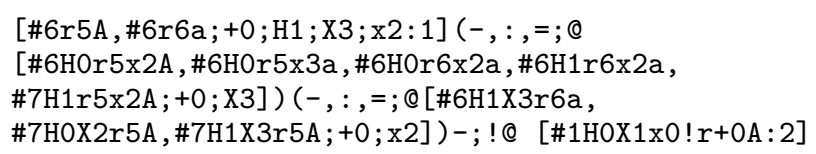 \\
\hline 320 & 1.410 & {$[*: 1] \sim[\# 8 \mathrm{H} 1: 2]$} \\
\hline 237 & 1.810 & {$[*: 1] \sim[\# 16: 2] \sim[\# 1]$} \\
\hline 274 & 1.336 & {$[\# 16 \mathrm{H} 1: 1](\sim[\# 6]) \sim[*: 2]$} \\
\hline 553 & 0.960 & {$[\# 8: 1] \sim[\# 1: 2]$} \\
\hline 317 & 1.495 & {$[*: 1](\sim[\# 6 \mathrm{X} 3 \mathrm{Ax} 2: 2]) \sim[*]$} \\
\hline 337 & 1.463 & {$[\# 6 \mathrm{H} 2: 1](\sim[\# 6 \mathrm{H} 2 \mathrm{x} 0]) \sim[\# 7: 2]$} \\
\hline 317 & 1.510 & {$[*: 1](\sim[\# 1])(\sim[*: 2]:[*])-[*]$} \\
\hline 570 & 1.229 & {$[*: 1](\sim[\# 8: 2]) \sim[* ; \mathrm{X} 3]$} \\
\hline 450 & 1.364 & {$[*: 1](\sim[\# 8: 2]) \sim ; @[*]$} \\
\hline 367 & 1.471 & {$[\# 7: 1](\sim[\# 1])(\sim[\# 1]) \sim[*: 2] \sim[\# 1]$} \\
\hline 317 & 1.504 & {$[*: 1](\sim[\# 6 \times 3 \times 2: 2] \sim[\# 7]) \sim[*]$} \\
\hline 447 & 1.419 & {$[*: 1](-[\# 6]) \sim[\# 6 \times 3: 2]$} \\
\hline 488 & 1.335 & {$[*: 1]=; @[*: 2]$} \\
\hline 422 & 1.385 & {$[*: 1](\sim[\# 6 \times 3 \times 2])-; @[*: 2]$} \\
\hline 512 & 1.375 & {$[*: 1](\sim[\# 7])=; @[*: 2] \sim[\# 1]$} \\
\hline 469 & 1.404 & {$[*: 1](-[\# 6])(:[\# 6 \times 3]) \sim[*: 2]$} \\
\hline 428 & 1.380 & {$[\# 7: 1] \sim[*: 2]:[*]$} \\
\hline 388 & 1.459 & {$[*: 1](\sim[*: 2]:[*])=[*]$} \\
\hline 469 & 1.409 & {$[*: 1]:[*: 2] \sim[\# 8]$} \\
\hline
\end{tabular}




\begin{tabular}{lll}
\hline & \multicolumn{2}{c}{ Polypeptide bond parameters } \\
\hline$k$ & $l$ & SMIRKS \\
\hline 477 & 1.343 & {$[*: 1] \sim ; @[*: 2]=[\# 7]$} \\
546 & 1.352 & {$[*: 1](\sim[\# 6 \times 3])=[*: 2]$} \\
410 & 1.394 & {$[\# 6 \times 2: 1] \sim[*: 2]=[\# 6]$} \\
427 & 1.381 & {$[*: 1](=[\# 6 \mathrm{H} 0]) \sim[*: 2](\sim[*]) \sim[*]$} \\
\hline
\end{tabular}

Table 5. These are the bond parameters from the reference force field with the associated SMIRKS patterns created with ChemPer

\begin{tabular}{|c|c|c|}
\hline \multicolumn{3}{|r|}{ Polypeptide angle parameters } \\
\hline$k$ & $\theta$ & SMIRKS \\
\hline 50 & 109.500 & {$[*: 1] \sim[*: 2] \sim[*: 3]$} \\
\hline 70 & 120.000 & 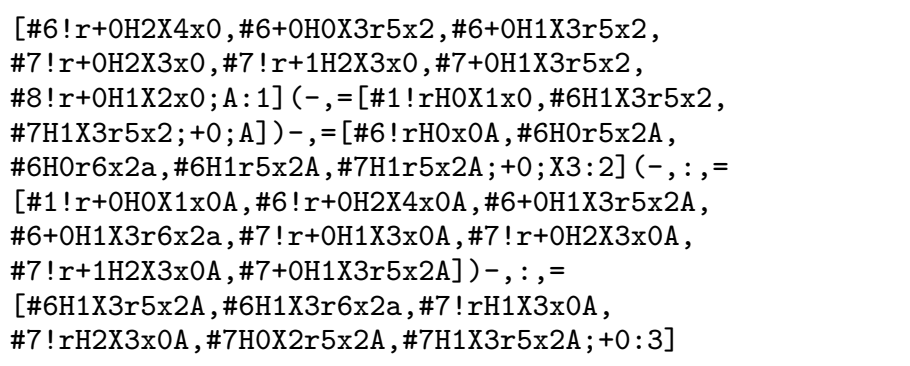 \\
\hline 50 & 120.000 & 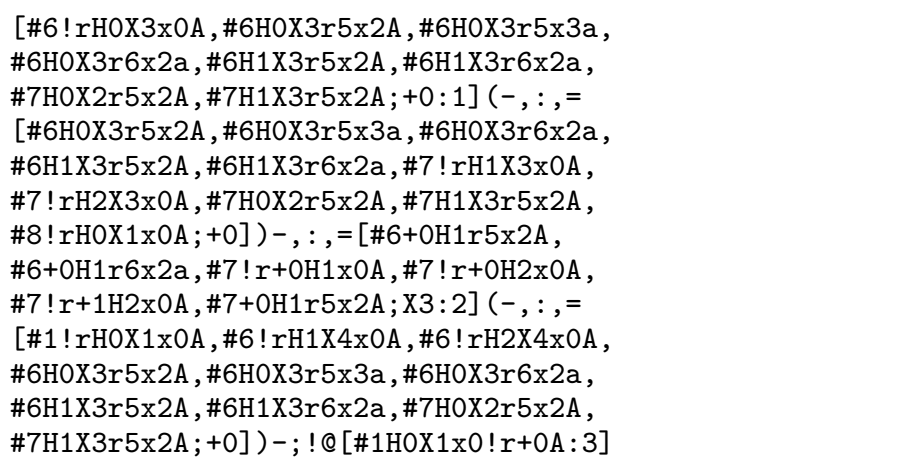 \\
\hline 40 & 109.5 & {$[\# 6: 1] \sim[* ; \mathrm{X} 4: 2] \sim[* ; \mathrm{X} 4: 3]$} \\
\hline 80 & 109.7 & {$[\# 7: 1]-[*: 2](\sim[\# 1])-[*: 3] \sim[*]$} \\
\hline 63 & 111.1 & {$[\# 6 ! r H 0: 1] \sim[*: 2] \sim[\# 6: 3]$} \\
\hline 70 & 116.6 & {$[\# 6: 1] \sim[\# 6 \mathrm{x} 0 \mathrm{H} 0: 2] \sim[*: 3]$} \\
\hline 50 & 121.9 & {$[*: 1](\sim[\# 8]) \sim[* ; \mathrm{X} 3: 2] \sim[\# 6: 3]$} \\
\hline 70 & 117.0 & {$[\# 6: 1] \sim[\# 6 ! r X 3, \# 7 \mathrm{X} 2: 2] \sim[* \mathrm{x} 2 \mathrm{H} 1, * \mathrm{xOH} 0: 3]$} \\
\hline 35 & 109.5 & {$[\# 1: 1] \sim[*: 2] \sim[\# 1: 3]$} \\
\hline 63 & 120.0 & {$[*: 1](\sim[*: 2] \sim ; @[*: 3]) \sim[*] \sim[*] \sim ; @[*]$} \\
\hline 63 & 110.1 & {$[\# 7: 1] \sim[*: 2] \sim[\# 6 \mathrm{xOHO}: 3]$} \\
\hline 80 & 120.4 & {$[\# 6: 1] \sim[*: 2](\sim[\# 7])=; ! @[*: 3]$} \\
\hline 80 & 122.9 & {$[\# 8: 1]=[*: 2](\sim[\# 6]) \sim[*: 3]$} \\
\hline 80 & 111.2 & $\begin{array}{l}{[\# 7: 1] \sim[\# 6 \mathrm{H} 2+0: 2] \sim[*: 3] \sim[*] \sim[*](\sim[\# 1])(\sim} \\
[* \mathrm{X} 1, * \mathrm{X} 3]) \sim[*] \sim[\# 7]\end{array}$ \\
\hline 50 & 118.040 & {$[*: 1](\sim[\# 7: 2](\sim[*: 3]) \sim[*] \sim[*] \sim[\# 7])(\sim[*]=; ! @[*]) \sim[*]$} \\
\hline 35 & 120.0 & {$[\# 1: 1] \sim[\# 7: 2](\sim[\# 1: 3]) \sim[*] \sim[\# 7, \# 8]$} \\
\hline 55 & 108.5 & {$[*: 1] \sim[\# 8: 2] \sim[*: 3]$} \\
\hline
\end{tabular}




\begin{tabular}{|c|c|c|}
\hline \multicolumn{3}{|r|}{ Polypeptide angle parameters } \\
\hline$k$ & $\theta$ & SMIRKS \\
\hline 50 & 114.7 & {$[*: 1](\sim[*: 2] \sim[\# 16: 3]) \sim[*]$} \\
\hline 50 & 108.6 & {$[*: 1](\sim[*: 2] \sim[\# 16: 3])(\sim[*] \sim[*]) \sim[*] \sim[*]$} \\
\hline 43 & 96.0 & {$[*: 1] \sim[\# 16: 2] \sim[*: 3]$} \\
\hline 62 & 98.9 & {$[*: 1](\sim[\# 16: 2] \sim[*: 3] \sim[*]) \sim[*]$} \\
\hline 63 & 113.1 & $\begin{array}{l}{[*: 1](\sim[*: 2] \sim[\# 6: 3](\sim ; @[*]) \sim[*] \sim[*]) \quad(\sim[*] \sim[*]) \sim[*] \sim} \\
{[*]}\end{array}$ \\
\hline 50 & 118.4 & {$[*: 1](\sim[*: 2](\sim[*: 3]) \sim[*]=; ! @[*] \sim[*]) \sim[*]$} \\
\hline 80 & 126.0 & {$[*: 1] \sim ; ! @[*: 2](-[\# 8: 3]) \sim[*] \sim[*] \sim[*] \sim[* \mathrm{H} 2 \mathrm{X} 4, * \mathrm{X} 3 \mathrm{H} 1]$} \\
\hline 63 & 115.6 & $\begin{array}{l}{[*: 1](\sim[*: 2] \sim[*: 3](\sim[*] \sim[*] \sim ; @[*]) \sim} \\
[*] \sim ; @[*])(\sim[*]-[*](\sim[\# 8]) \sim[*] \sim[*])-[*] \sim[*] \sim[*] \sim[*]\end{array}$ \\
\hline 50 & 123.2 & {$[*: 1](\sim[*: 2] \sim[*: 3](\sim[\# 7]) \sim[\# 7]) \sim[*]$} \\
\hline 50 & 113.0 & {$[*: 1](\sim[\# 8: 2] \sim[*: 3]) \sim[*] \sim[*] \sim ; @[*]$} \\
\hline 63 & 114.0 & $\begin{array}{l}{[*: 1](-[*: 2] \sim[*: 3](:[*]) \sim[*] \sim[*] \sim[*] \sim} \\
[*])(\sim[*] \sim[*] \sim[*]) \sim ; ! \Theta[*] \sim[*] \sim[*] \sim[\# 1]\end{array}$ \\
\hline 70 & 128.6 & {$[*: 1](\sim[\# 6: 2](-[\# 6: 3]:[*] \sim[*]) \sim ; @[*]) \sim[*]$} \\
\hline 70 & 125.0 & $\begin{array}{l}{[\# 6 \mathrm{H} 2: 1](\sim[*: 2](\sim[*: 3]) \sim[*](\sim ; @[*]) \sim[*] \sim[*])} \\
\sim[*] \sim[*] \sim[*]\end{array}$ \\
\hline 50 & 123.1 & {$[*: 1](\sim[*: 2] \sim[*: 3])(:[*]-[\# 6]):[*]$} \\
\hline 70 & 132.8 & {$[*: 1](-[*: 2]:[*: 3]) \sim[*] \sim ; @[*]$} \\
\hline 63 & 122.7 & {$[*: 1](\sim[*: 2]:[*: 3])(-[*] \sim[*] \sim[\# 6] \sim[\# 1]) \sim[*] \sim[\# 1]$} \\
\hline 63 & 108.8 & $\begin{array}{l}{[*: 1](\sim[*: 2](:[*: 3]) \sim[*] \sim[*] \sim[*])(-[*](\sim} \\
[*]) \sim[*]) \sim[*] \sim[*]\end{array}$ \\
\hline 50 & 118.0 & $\begin{array}{l}{[*: 1](\sim[*: 2](\sim[*: 3]) \sim[*] \sim ; ! @[*] \sim[*])} \\
(\sim ; ! @[*])(\sim ; ! @[*] \sim[*] \sim[*]) \sim ; @[*]\end{array}$ \\
\hline 63 & 116.2 & $\begin{array}{l}{[*: 1](\sim[*: 2]:[*: 3])(\sim[*]-; @[[*](\sim[*]) \sim[*])} \\
\sim[*](\sim[*]) \sim[*]\end{array}$ \\
\hline 70 & 111.6 & {$[*: 1](\sim[\# 7: 2] \sim[*: 3]:[*]) \sim[*]$} \\
\hline 70 & 108.7 & $\begin{array}{l}{[*: 1](\sim[*: 2](\sim[*: 3]) \sim ; ! @[*])(\sim[*] \sim[*] \sim} \\
[*] \sim[*]) \sim[*](\sim[*]:[*]):[*]\end{array}$ \\
\hline 70 & 104.4 & $\begin{array}{l}{[*: 1](\sim ; @[*: 2]-[*: 3] \sim[*])(\sim ; @[*])-[*]} \\
(\sim[*] \sim[*]) \sim[*] \sim[*]\end{array}$ \\
\hline 63 & 106.4 & {$[*: 1] \sim[*: 2](\sim[\# 6 \times 3: 3]) \sim[*](\sim[\# 1]) \sim[*] \sim[\# 7]$} \\
\hline 63 & 134.9 & $\begin{array}{l}{[*: 1](-[*: 2] \sim ; @[*: 3] \sim[*] \sim[*])(\sim[*] \sim[*]} \\
\sim ; @[*]) \sim[*] \sim[*] \sim[*] \sim[*]\end{array}$ \\
\hline
\end{tabular}

Table 6. These are the angle parameters from the reference force field with the associated SMIRKS patterns created with ChemPer

\begin{tabular}{|c|c|c|c|}
\hline \multicolumn{4}{|c|}{ Polypeptide improper torsion parameters } \\
\hline$n$ & $\omega$ & $u$ & SMIRKS \\
\hline 2 & 180 & 1.100 & 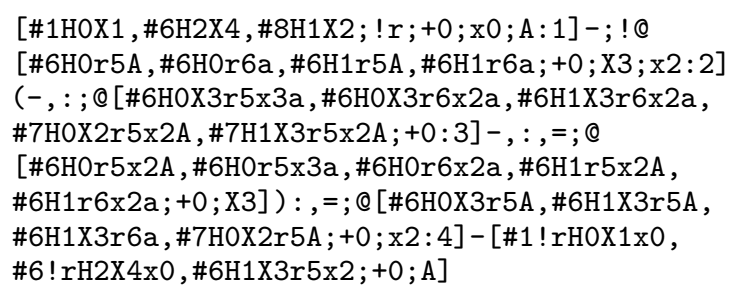 \\
\hline
\end{tabular}




\begin{tabular}{llll}
\hline \multicolumn{4}{c}{ Polypeptide improper torsion parameters } \\
\hline$n$ & $\omega$ & $u$ & SMIRKS \\
\hline 2 & 180 & 1.000 & {$[*: 1] \sim[\# 7: 2](\sim[*: 3]) \sim[*: 4]$} \\
2 & 180 & 10.500 & {$[*: 1] \sim ; ! @[\# 6: 2](\sim[*: 3]) \sim ; ! @[*: 4]$} \\
2 & 180 & 1.100 & {$[*: 1](\sim[\# 8]) \sim ; ! @[*: 2](\sim[*: 3] \sim[*]) \sim ; ! @[*: 4]$} \\
\hline
\end{tabular}

Table 7. These are the improper torsion parameters from the reference force field with the associated SMIRKS patterns created with ChemPer

\begin{tabular}{|c|c|c|c|}
\hline \multicolumn{4}{|c|}{ Polypeptide proper torsion parameters } \\
\hline$n$ & $\omega$ & $u$ & SMIRKS \\
\hline 3 & 0 & 0.156 & 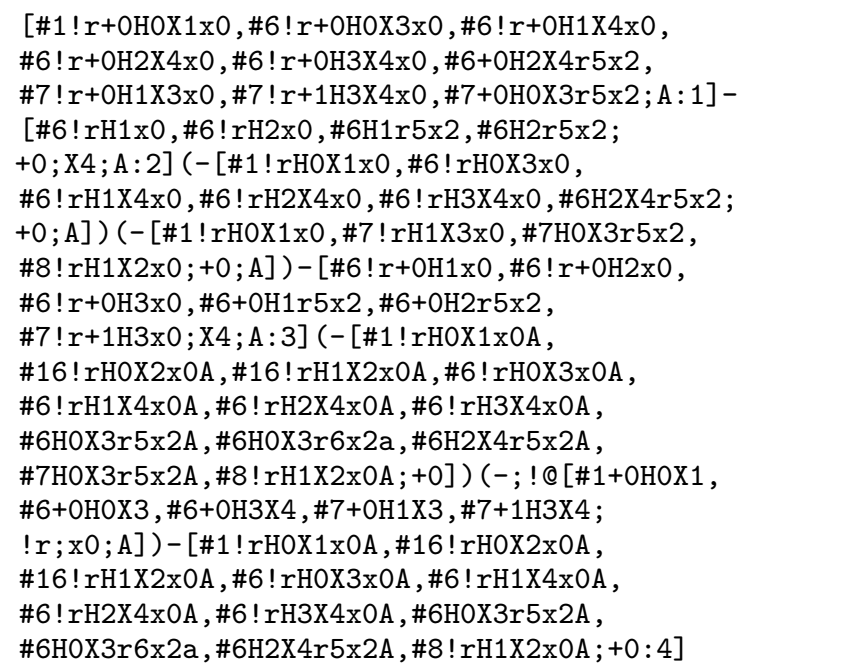 \\
\hline 2 & 180 & 3.625 & 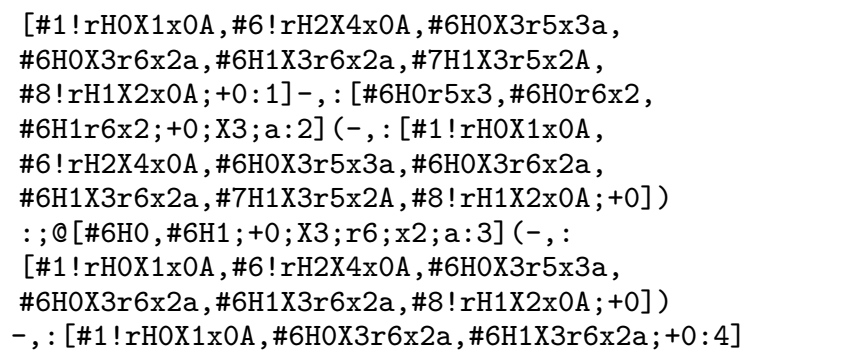 \\
\hline 2 & 180 & 2.400 & {$[*: 1] \sim[* ; \mathrm{X} 3 ; \mathrm{A}: 2] \sim[*: 3] \sim[*: 4]$} \\
\hline 2 & 180 & 2.500 & {$[\# 6, \# 8: 1] \sim[\# 6: 2] \sim[\# 7 \mathrm{H} 1, \# 7 \mathrm{H} 2 \mathrm{x} 0, \# 7 \mathrm{HO}: 3] \quad(\sim[*: 4]) \sim[*]$} \\
\hline 3 & 0 & 0.160 & $\begin{array}{l}{[\# 6, \# 6: 1](\sim[\# 1]) \sim[*: 2] \sim[\# 6: 3](\sim[\# 1: 4])} \\
(\sim[\# 1 \mathrm{X} 1, \# 6 \mathrm{X} 4]) \sim[* ! r \mathrm{X} 1 \mathrm{H} 0, * ! \mathrm{rX} 3 \mathrm{H} 0, * ! r \mathrm{X} 4 \mathrm{H} 1 \\
* \mathrm{H} 2 \mathrm{X} 4, * ! r \mathrm{HXX} 4, * \mathrm{X} 4 \mathrm{r} 5, * \mathrm{H} 2 \mathrm{X} 4 \mathrm{r} 5 ;+0]\end{array}$ \\
\hline 3 & 0 & 0.180 & {$[* ;+0 ; \mathrm{X} 4: 1] \sim[* ; \mathrm{X} 4: 2] \sim[*: 3] \sim[\# 6 \mathrm{H} 3, \# 6 \mathrm{H} 2: 4]$} \\
\hline 2 & 180 & 0.250 & \\
\hline 1 & 180 & 0.200 & \\
\hline 3 & 0 & 0.400 & {$[*: 1] \sim[\# 6: 2] \sim[\# 6 \mathrm{X} 3 \mathrm{~A}: 3] \sim[*: 4]$} \\
\hline 2 & 0 & 0.200 & \\
\hline 1 & 0 & 0.200 & \\
\hline 3 & 0 & 0.400 & {$[*: 1](\sim[\# 7: 2] \sim[*: 3] \sim[*: 4])=[*]$} \\
\hline 2 & 0 & 2.000 & \\
\hline 1 & 0 & 2.000 & \\
\hline
\end{tabular}


Preprint ahead of submission - June 21, 2019

Polypeptide proper torsion parameters

\begin{tabular}{|c|c|c|c|}
\hline$n$ & $\omega$ & $u$ & SMIRKS \\
\hline 3 & 180 & 0.550 & {$[* ; \mathrm{X} 3: 1] \sim[*: 2] \sim[\# 6 \mathrm{X} 3 \mathrm{x} 0: 3] \sim[*: 4]$} \\
\hline 2 & 180 & 1.580 & \\
\hline 1 & 180 & 0.450 & \\
\hline 3 & 0 & 0.150 & $\begin{array}{l}{[\# 1: 1] \sim[*: 2](\sim[\# 6])(\sim[* ;+0: 3](\sim[\# 1, \# 6, \# 6,} \\
\# 6])(\sim[\# 1: 4]) \sim[\# 1]) \sim[* \mathrm{X} 1 \mathrm{HO}, * \mathrm{X} 4 \mathrm{H} 1, * \mathrm{H} 3 \mathrm{X} 4]\end{array}$ \\
\hline 3 & 0 & 0.333 & {$[*: 1] \sim[\# 16: 2] \sim[*: 3] \sim[*: 4]$} \\
\hline 3 & 0 & 0.420 & {$[*: 1](\sim[\# 7: 2] \sim[*: 3] \sim[\# 6 ! \mathrm{rHO}: 4]) \sim[*]$} \\
\hline 2 & 0 & 0.270 & \\
\hline 2 & 180 & 3.000 & 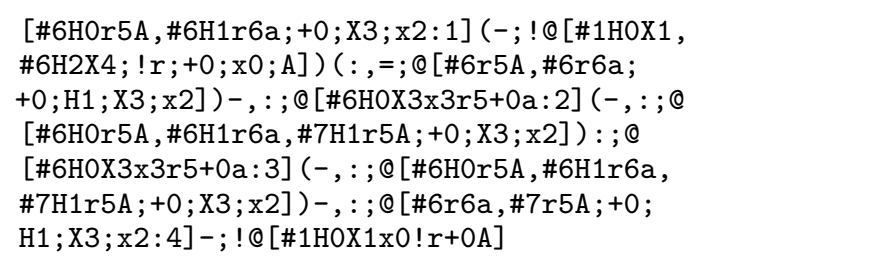 \\
\hline 1 & 0 & 0.800 & {$[*: 1]=[*: 2] \sim ; ! @[\# 6: 3] \sim[*: 4]$} \\
\hline 3 & 180 & 0.080 & \\
\hline 1 & 0 & 0.250 & {$[\# 8: 1]-[*: 2]-[*: 3] \sim[\# 1: 4]$} \\
\hline 3 & 0 & 0.160 & {$[*: 1] \sim[*: 2] \sim[\# 8: 3] \sim[*: 4]$} \\
\hline 1 & 0 & 0.250 & \\
\hline 2 & 180 & 3.500 & {$[\# 6: 1](\sim[\# 6 \mathrm{X} 3 \mathrm{r} 5: 2] \sim[\# 6 \times 2: 3] \sim[*: 4]) \sim[* r 5 \mathrm{H} 1]$} \\
\hline 2 & 180 & 1.675 & {$[\# 6: 1]-,=[*: 2](\sim[\# 6 \mathrm{X} 4 \mathrm{x} 0, \# 6 \mathrm{X} 3 \mathrm{r} 5 \mathrm{x} 2]) \sim ; @[*: 3] \sim[*: 4]$} \\
\hline 2 & 180 & 2.325 & {$[*: 1]-[*: 2] \sim[\# 6 \mathrm{X} 3 \mathrm{r} 5 \mathrm{H} 1: 3] \sim[*: 4]$} \\
\hline 2 & 180 & 1.500 & {$[*: 1] \sim[\# 6 \mathrm{X} 3 \mathrm{H} 1: 2]-[*: 3](\sim[* \mathrm{x} 0, * \mathrm{x} 3: 4]) \sim[* \mathrm{x} 0, * \mathrm{x} 3]$} \\
\hline 2 & 180 & 1.525 & {$[*: 1] \sim[*: 2](\sim[\# 7: 3] \sim[*: 4]):[*]$} \\
\hline 2 & 180 & 1.400 & $\begin{array}{l}{[\# 6 ! r, \# 6 r 5 \times 2 \mathrm{H} 1: 1](\sim[\# 1])(\sim[*: 2]-; @[*: 3]} \\
-[*: 4]) \sim[* \mathrm{X} 1, * \mathrm{X} 2]\end{array}$ \\
\hline 2 & 180 & 6.525 & {$[*: 1]-[\# 6 \times 3: 2](\sim[\# 6: 3] \sim[*: 4])-[*]$} \\
\hline 2 & 180 & 5.150 & {$[* ; \mathrm{A}: 1] \sim[*: 2](\sim ; @[\# 6: 3] \sim[\# 1, \# 7: 4]) \sim[\# 6 \mathrm{H} 2 \mathrm{x} 0, \# 7 \mathrm{x} 2 \mathrm{H} 1]$} \\
\hline 6 & 180 & 0.013 & {$[\# 6: 1] \sim[\# 6: 2] \sim[*: 3] \sim[\# 8 ; \mathrm{X} 1: 4]$} \\
\hline 4 & 180 & 0.138 & \\
\hline 2 & 180 & 0.443 & \\
\hline 3 & 0 & 0.250 & {$[*: 1] \sim[*: 2] \sim[\# 16: 3] \sim[\# 1: 4]$} \\
\hline 2 & 180 & 2.500 & {$[\# 8: 1] \sim[*: 2] \sim[\# 7: 3] \sim[\# 1: 4]$} \\
\hline 1 & 0 & 2.000 & \\
\hline 3 & 0 & 0.167 & {$[\# 1: 1] \sim[*: 2] \sim[\# 8: 3] \sim[*: 4]$} \\
\hline 2 & 180 & 5.000 & {$[\# 6: 1](\sim[*: 2]=[*: 3] \sim[*: 4])=[*]$} \\
\hline 6 & 180 & 0.101 & {$[*: 1](\sim[*: 2](\sim[\# 7]) \sim[*: 3] \sim[\# 6 \mathrm{x} 0: 4] \sim[\# 7]) \sim[*]$} \\
\hline 5 & 0 & 0.104 & \\
\hline 4 & 180 & 0.417 & \\
\hline 3 & 0 & 0.118 & \\
\hline 2 & 180 & 0.596 & \\
\hline 1 & 0 & 0.571 & \\
\hline
\end{tabular}


Preprint ahead of submission - June 21, 2019

\begin{tabular}{llll}
\hline & \multicolumn{3}{c}{ Polypeptide proper torsion parameters } \\
\hline$n$ & $\omega$ & $u$ & SMIRKS \\
\hline 3 & 0 & 0.135 & {$[*: 1](\sim[\# 6 \mathrm{H} 1: 2] \sim[*: 3] \sim[\# 6 \mathrm{H} 1: 4])=[*]$} \\
2 & 180 & 0.358 & \\
1 & 0 & 0.571 & \\
2 & 180 & 0.846 & {$[*: 1](\sim[\# 6 \mathrm{AHO}]) \sim[*: 2] \sim[*: 3](\sim[\# 6 \mathrm{H} 2]) \sim[*: 4] \sim[*]$} \\
1 & 0 & 0.195 & \\
6 & 180 & 0.213 & {$[*: 1](\sim[*: 2](\sim[\# 6]) \sim[*: 3] \sim[*: 4](\sim[\# 8]) \sim[\# 8]) \sim[*]$} \\
5 & 0 & 0.232 & \\
4 & 0 & 0.423 & \\
3 & 180 & 0.007 & \\
2 & 180 & 1.190 & \\
1 & 180 & 2.635 & \\
6 & 180 & 0.106 & {$[*: 1](\sim ; ! @[\# 7]) \sim[*: 2] \sim[\# 6 \mathrm{HO}: 3] \sim[*: 4] \sim[*]$} \\
5 & 0 & 0.130 & \\
4 & 0 & 0.100 & \\
3 & 180 & 0.035 & \\
2 & 180 & 0.181 & \\
1 & 180 & 1.046 & \\
3 & 180 & 0.080 & {$[*: 1]=[*: 2] \sim[*: 3](\sim[\# 1])(\sim[\# 6 \mathrm{H} 1]) \sim[*: 4]$} \\
1 & 0 & 0.800 & \\
2 & 180 & 2.300 & \\
\hline
\end{tabular}

Table 8. These are the proper torsion parameters from the reference force field with the associated SMIRKS patterns created with ChemPer

\begin{tabular}{lll}
\hline & \multicolumn{2}{c}{ Polypeptide Lennard-Jones parameters } \\
\hline$\epsilon$ & $r_{\min }$ & SMIRKS \\
\hline 0.170 & 1.824 & {$[*: 1]$} \\
0.086 & 1.908 & {$[* ;+0 ; \mathrm{X} 3: 1]:,=[*]$} \\
0.109 & 1.908 & {$[\# 6 ; \mathrm{X} 4: 1]$} \\
0.250 & 2.000 & {$[* ; \mathrm{X} 2 ; ! \mathrm{r}: 1]$} \\
0.210 & 1.661 & {$[\# 8: 1]$} \\
0.016 & 1.100 & {$[\# 1: 1]$} \\
0.015 & 1.459 & {$[\# 1: 1] \sim[*] \sim[\# 6 \mathrm{x} 2]$} \\
0.015 & 1.409 & {$[\# 1: 1] \sim[*]=[*]$} \\
0.016 & 1.487 & {$[\# 1: 1] \sim[*](\sim[* ;+0])(\sim[* ;+0]) \sim[* ;+0]$} \\
0.000 & 0.000 & {$[\# 1: 1] \sim[\# 8]$} \\
0.210 & 1.721 & {$[\# 8: 1] \sim[\# 1]$} \\
0.015 & 1.359 & {$[*: 1] \sim ; ! @[*]=; @[\# 7]$} \\
0.016 & 1.387 & {$[\# 1: 1] \sim[*] \sim[\# 16, \# 7 \mathrm{X} 3 \mathrm{x} 0, \# 7 \mathrm{X} 3 \mathrm{H} 0, \# 8]$} \\
0.016 & 0.600 & {$[\# 1: 1] \sim[\# 16, \# 7]$} \\
\hline
\end{tabular}

Table 9. These are the Lennard-Jones parameters from the reference force field with the associated SMIRKS patterns created with ChemPer 
Other files

electronic_SI.zip (46.32 MiB)

view on ChemRxiv - download file 